\title{
QCD rescattering mechanism for diffractive deep inelastic scattering
}

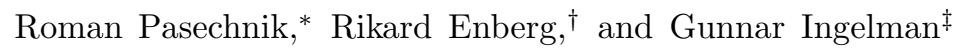

Department of Physics and Astronomy, Uppsala University, Box 516, SE-751 20 Uppsala, Sweden

\begin{abstract}
We present a QCD-based model where rescattering between final state partons in deep inelastic scattering leads to events with large rapidity gaps and a leading proton. In the framework of this model the amplitude for multiple gluon exchanges is calculated in the eikonal approximation to all orders in perturbation theory. Both large and small invariant mass $M_{X}$ limits are considered. The model successfully describes the precise HERA data on the diffractive deep inelastic cross section in the whole available kinematical range and gives new insight into the density of gluons at very small momentum fractions in the proton.
\end{abstract}

\section{INTRODUCTION}

Hadronic processes with a hard scale involved constitute an indispensable tool for probing the QCD dynamics of quarks and gluons, and through the QCD factorization theorems [1] that separate physics at small and large distances, one may also study the dynamics of soft processes with small momentum transfers. Hard quark and gluon interactions at small distances are thus not affected by soft interactions and are described in perturbative QCD. The most problematic part of the process is soft interactions at large distances, where nonperturbative QCD comes into the game and manifests itself as the confinement of quarks and gluons in hadrons and the related hadronization process giving the observable hadronic final states in high energy collisions.

Diffractive processes are sensitive to the details of nonperturbative QCD dynamics and provide a way to probe the soft and semihard regimes directly. Diffractive events are characterized by a leading "target" particle, carrying most of the beam momentum, and a well separated produced hadronic system. The "gap" in between is connected to the soft part of the event and therefore to nonperturbative effects at a long space-time scale. Diffractive deep inelastic scattering (DDIS) offers a particularly good opportunity to explore the interplay between hard and soft physics due to the precise data from the electron-proton collider HERA [2, 3].

DDIS in lepton-proton collisions involves hard scattering events where, in spite of the large momentum transfer $Q^{2}$ from the electron, the proton emerges essentially unscathed with small transverse momentum, keeping almost all of its original longitudinal beam momentum (for reviews on DDIS, see e.g. Refs. [4 6$]$ ). The leading proton is well separated in momentum space, or rapidity $y=1 / 2 \ln \left(E+p_{z}\right) /\left(E-p_{z}\right)$, from the central hadronic system produced from the exchanged virtual photon's interaction with the proton. Thus, this new class of events

\footnotetext{
*Electronic address: roman.pasechnik@fysast.uu.se

${ }^{\dagger}$ Electronic address: rikard.enberg@physics.uu.se

‡Electronic address: gunnar.ingelman@physics.uu.se
}

is characterized by a large rapidity gap (LRG) void of final state particles.

Rapidity gaps in deep inelastic scattering (DIS) were discovered by the ZEUS and H1 experiments at HERA [3], but the first discovery of hard diffraction was in $p \bar{p}$ collisions by the UA8 experiment 7]. These processes had actually been predicted [8] by combining Regge phenomenology for soft processes in strong interactions via pomeron exchange, with hard processes based on perturbative QCD. By parametrizing the parton content of an exchanged pomeron (or alternatively diffractive parton density functions) it is possible to describe the HERA data. However, the extracted parton densities are not universal, since when used to calculate diffractive hard scattering processes in $p \bar{p}$ collisions at the Tevatron one obtains cross sections an order of magnitude larger than observed.

An alternative dynamical interpretation of hard diffraction was proposed in Refs. [9]. This Soft Color Interaction (SCI) model is based on the simple assumption of soft gluon exchanges leading to color rearrangements between the final state partons. Variations in the topology of the confining color fields lead to different hadronic final states.

The SCI model is implemented in Monte Carlo event generators, e.g. LEPTO for DIS [10]. The hard part of the process shown in Fig. 1 is then calculated in the framework of perturbative QCD with DGLAP evolution

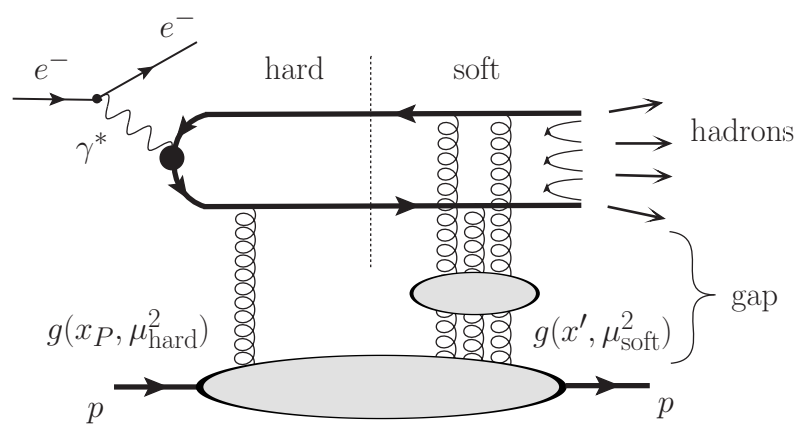

FIG. 1: Schematic illustration of the mechanism for diffractive deep inelastic scattering considered in this paper, with soft gluon exchanges in the final state. 
of the parton showers in the same way as in inclusive DIS. The large momentum transfer means that the hard subprocess occurs on a spacetime scale much smaller than the bound state proton and is thus "embedded" in the proton. The emerging hard-scattered partons propagate through the proton's color field and may interact with it. Soft exchanges will dominate due to the large coupling and the lack of suppression from hard gluon propagators. Therefore, the momenta of the hard partons are essentially undisturbed - the soft, long distance interactions do not affect the hard, short distance process, and the momentum transfer of the soft exchanges can be neglected. However, the exchange of color changes the color charges of the emerging partons such that the confining string-like field between them will have a different topology, resulting in a different distribution of final state hadrons produced from the string hadronization [11]. In particular, a region in rapidity without a string will result in an absence of hadrons there, i.e. a rapidity gap.

The only parameter of this model is the probability for a soft exchange, accounting for the unknown nonperturbative dynamics. Remarkably, the SCI model is phenomenologically very successful in describing many different processes, both diffractive and nondiffractive [12], with only a single parameter $P \simeq 0.5$ for this probability. Thus, the SCI model captures the essential dynamics of diffraction, but lacks a solid theoretical basis.

To understand better what we can learn from the phenomenology of the SCI model, we present in this paper a detailed QCD-based mechanism for soft gluon rescattering of final state partons, as illustrated in Fig. 1] This mechanism leads to effective color singlet exchange and thereby to diffractive scattering. Inspired by the SCI model, the model presented here may be seen as an explicit realization of the earlier attempt [13] to understand soft gluon exchange in terms of QCD rescattering. Our model was initially introduced in a recent letter [14], and is here presented in detail.

The paper is organized as follows. In Section II we briefly discuss the framework of the dipole approach and motivate our study. In Section III we consider the kinematics of diffractive DIS. Section IV treats the formalism for generalized unintegrated gluon distribution functions in the diffractive limit. The explicit calculation of the $q \bar{q}$ dipole contribution to the diffractive cross section and analytic approximations used are presented in Section V. In Section VI we study the contribution of the $q \bar{q} g$ final state. Numerical results and comparisons with HERA data on the diffractive cross section are given in Section VII. Finally, in Section VIII we present some concluding remarks and an outlook.

\section{DIPOLE APPROACH}

Typical contributions to the diffractive DIS process are represented by the diagrams in Fig. 2. In terms of the four-momenta $q$ of the photon, and $P$ and $P^{\prime}$ of the ini-
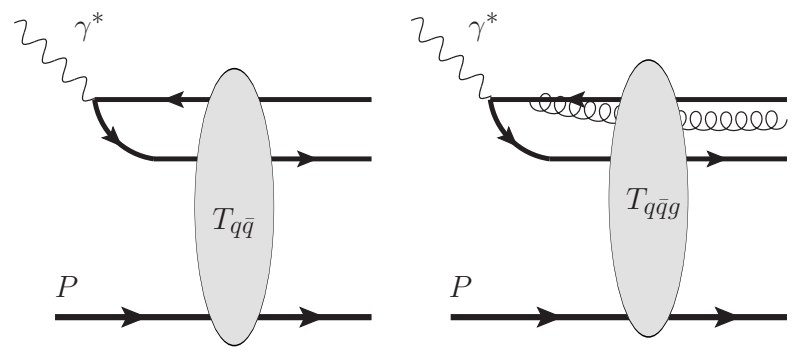

FIG. 2: Typical diagrams contributing to diffractive DIS with the leading order $q \bar{q}$ dipole scattering (left) and the gluonic $q \bar{q} g$ contribution (right) contributing significantly at $\beta \rightarrow 0$ (large $\left.M_{X}\right)$.

tial and final proton, the kinematics of the $\gamma^{*} P \rightarrow X P^{\prime}$ process is defined by the variables

$$
x_{B}=\frac{Q^{2}}{Q^{2}+W^{2}}, \quad \beta=\frac{Q^{2}}{Q^{2}+M_{X}^{2}}, \quad x_{P}=\frac{x_{B}}{\beta},
$$

where $Q^{2}=-q^{2}$. The invariant mass of the produced system $M_{X}$, and the total energy in the $\gamma^{*} P$ center-ofmass system $W$ are given by

$$
M_{X}^{2}=\frac{1-\beta}{\beta} Q^{2}, \quad W^{2} \equiv(P+q)^{2}=\frac{Q^{2}}{x_{B}}\left(1-x_{B}\right) .
$$

The DDIS cross section in general is represented as a function of $\beta, x_{P}, Q^{2}$ and the momentum transfer along the proton line $t=\left(P^{\prime}-P\right)^{2}$. Note, that we are working in the forward limit of small $|t| \ll Q^{2}, M_{X}^{2}$.

Let us consider first the simplest case of the $q \bar{q}$ contribution, which is the leading one for small $M_{X}$ (or, equivalently, $\beta \rightarrow 1$ ). To compute the diffractive DIS amplitude, it is convenient to consider the process in the dipole frame [15], where the deeply virtual photon with large virtuality $Q^{2}$ and polarization $\lambda$ first splits into a quark $q$ and an antiquark $\bar{q}$ with mass $m_{q}$, spins $\alpha$ and $\beta$, and flavor $f$, and then the $q \bar{q}$ dipole with transverse size $r$ interacts with the target proton at impact parameter $b$ and dissociates into a final state $X$ of invariant mass $M_{X}$ as shown in Fig. 3. The photon splitting into the dipole is a QED process and is described by the wavefunctions $\psi_{\lambda}^{f, \alpha \beta}\left(z, \mathbf{r} ; Q^{2}\right)$ in the impact parameter space [15, 16], where $z$ is the fraction of the longitudinal momentum carried by the quark. The amplitude of the dipole-nucleon scattering (denoted as $T$ in Fig. 2) is the only unknown non-perturbative object (for a review, see Ref. [5]). The dipole picture naturally incorporates the description of both inclusive and diffractive events into a common theoretical framework [16, 17], as the same dipole scattering amplitudes enter into inclusive and diffractive cross sections.

Final states with gluons are suppressed by powers of $\alpha_{s}$. However, if $\beta$ becomes small or $Q^{2}$ large, the $q \bar{q}$ dipole emits soft or collinear gluons accompanied by large logarithms $\ln (1 / \beta)$ or $\ln Q^{2}$ which compensate the 
suppression in $\alpha_{s}$ [18]. The $q \bar{q}$ pair can emit soft gluons, leading to the dressing up of the quarks, which is parametrized by a scale-dependent constituent quark mass $m_{q}^{\text {eff }}\left(\mu^{2}\right)$. In general, the more gluons in the final state, the larger the invariant mass produced. The dominant gluon emission from quarks is described by DGLAP evolution [19] and is mostly collinear to the radiating quark, so it cannot build up a large $M_{X}$. The small $\beta \rightarrow 0$ and large $Q^{2} \rightarrow \infty$ limits can be driven, therefore, only by a semihard gluon radiation from the active gluon (carrying $x_{P}$ ) giving rise to a gluonic dipole $q \bar{q} g$ contribution. These aspects will be discussed in detail below.

Diffractive DIS at the leading order in $\alpha_{s}$ is described by the two gluon exchange diagram shown in Fig. 3 . Let us first discuss how the longitudinal momentum transfer between the $q \bar{q}$ dipole and the proton can be shared between the gluons. The gluon momenta can be Sudakov decomposed as

$$
q_{1}=-x P+\Delta_{\perp}, \quad q_{2}=-x^{\prime} P+\Delta_{\perp}^{\prime}, \quad x+x^{\prime}=x_{P} .
$$

Cutting the diagram after the first gluon exchange (picking up the leading poles only), in the high-energy limit $x_{B} \rightarrow 0$, we have

$$
\left(q+x P-\Delta_{\perp}\right)^{2}=M_{\mathrm{int}}^{2} \rightarrow-2 P q\left(x_{B}-x\right)=M_{\mathrm{int}, \perp}^{2}
$$

In the deep inelastic limit $Q^{2} \rightarrow \infty$, for fixed invariant mass of the intermediate system $M_{\mathrm{int}} \sim M_{X}$ and Bjorken variable $x_{B}$, we see that $M_{\mathrm{int}}^{2} \ll 2 P q$ and $x \simeq x_{B}$, thus $x^{\prime}=x_{P}-x_{B}$. On the other hand, when $Q^{2} \rightarrow \infty$ and $M_{\text {int }}^{2}$ fixed, we see that $\beta=x_{B} / x_{P} \rightarrow 1$. So $x \rightarrow x_{P}$ and $x^{\prime} \rightarrow 0$, and the first gluon takes all the longitudinal momentum exchange neutralizing the virtuality of the $q \bar{q}$ system. The latter kinematical configuration gives the leading contribution to the cross section, whereas the other configurations with equal momentum sharing between the gluons $x \sim x^{\prime} \sim x_{P} / 2$ are suppressed by extra propagators.

Thus, we consider the asymmetric case with one hard (perturbative) gluon carrying most of the longitudinal momentum transfer $x_{P}$, and a number of multiple soft screening interactions with total $x^{\prime} \ll x_{P}$ in a color octet

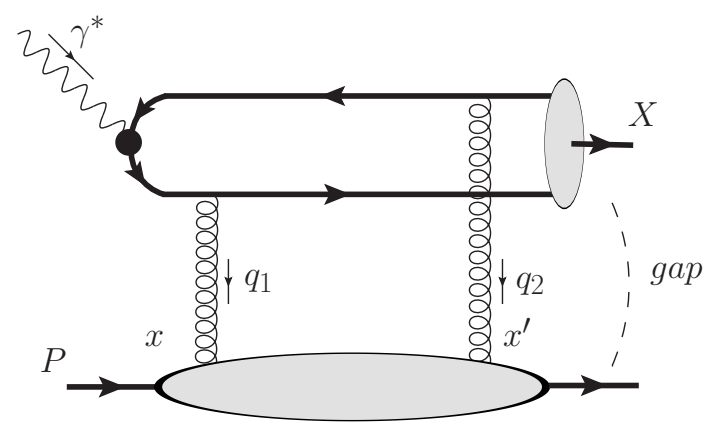

FIG. 3: Amplitude of the process $\gamma^{*} p \rightarrow X p$ at the leading order of perturbation theory.

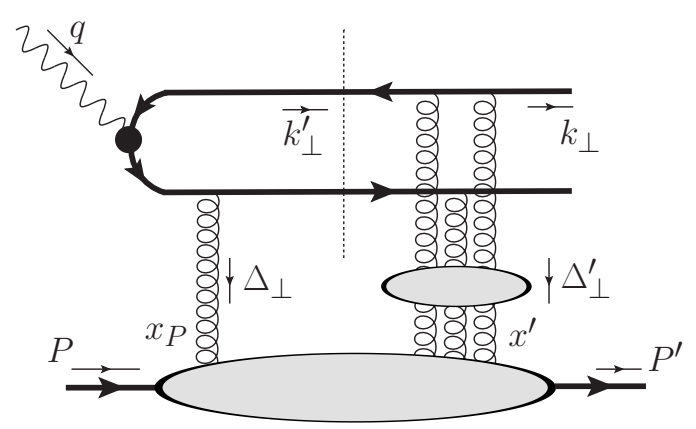

FIG. 4: Amplitude of the process $\gamma^{*} p \rightarrow X p$ with all-order resummed soft gluon exchange.

state (in the large $N_{c}$ limit) effectively described by the resummed multigluon exchange amplitude as schematically sketched in Fig. 4. The first "hard" gluon turns the proton into a color octet proton remnant, which then interacts with a system of soft screening gluons in an octet state and finally recombines into a color singlet corresponding to the leading proton (or system with invariant mass close to the proton mass). These soft gluons cannot dynamically resolve a $q \bar{q}$ dipole of small size $r \sim 1 / k_{\perp}$, However, we assume that the gluons interact with the quark current and not with the dipole as a whole. This will be further discussed below in Sec. VE.

\section{KINEMATICS OF DIFFRACTIVE DIS}

Let us first define the kinematics of the process $\gamma^{*} p \rightarrow$ $X p$. Our primary interest is processes with small momentum transfer $t \ll Q^{2}$. It is convenient to fix the frame of reference in the center-of-mass system of the final states, i.e. the outgoing proton with momentum $P^{\prime}$, and the diffractive system $X$ with momentum $q^{\prime}=k_{1}+k_{2}$, $\mathbf{k}_{1, \perp}=-\mathbf{k}_{2, \perp} \equiv \mathbf{k}_{\perp}$.

The total $\gamma^{*} p$ c.m.s. energy squared is $W^{2}=\left(P^{\prime}+\right.$ $\left.q^{\prime}\right)^{2}=2 P^{\prime} q^{\prime}+M_{X}^{2}$ (the proton mass $m_{p}$ is neglected). In terms of the cross section variables defined in Eqs. (1) and (2) we write

$2 P^{\prime} q^{\prime}=W^{2}-M_{X}^{2}=Q^{2} \frac{1-x_{P}}{x_{B}}, \quad 2 P q=\frac{Q^{2}}{x_{B}} \simeq 2 P^{\prime} q^{\prime}$.

Since $x_{P} \ll 1$, we have $W^{2} \gg M_{X}^{2}$ for any $\beta$ and $Q^{2}$, so we will first keep $M_{X}^{2}$ and then drop it in comparison with $W^{2}$ whenever appropriate.

The general Sudakov decompositions of the final quark/antiquark momenta $k_{1,2}$ are

$k_{1}=(1-z) q^{\prime}+n_{1} P^{\prime}+k_{1, \perp}, \quad k_{2}=z q^{\prime}+n_{2} P^{\prime}+k_{2, \perp}$.

where $n_{1}=-n_{2}$. The on-shell conditions for the quark and antiquark in the final state

$$
\begin{gathered}
(1-z)^{2} M_{X}^{2}+n_{1}(1-z)\left(W^{2}-M_{X}^{2}\right)-k_{\perp}^{2}=m_{q}^{2}, \\
z^{2} M_{X}^{2}+n_{2} z\left(W^{2}-M_{X}^{2}\right)-k_{\perp}^{2}=m_{q}^{2}
\end{gathered}
$$


give

$$
n_{1}=\frac{m_{q, \perp}^{2}-(1-z)^{2} M_{X}^{2}}{(1-z)\left(W^{2}-M_{X}^{2}\right)}, \quad n_{2}=\frac{m_{q, \perp}^{2}-z^{2} M_{X}^{2}}{z\left(W^{2}-M_{X}^{2}\right)} .
$$

Finally, $n_{2}=-n_{1} \equiv n$ leads to

$$
M_{X}^{2}=\frac{m_{q, \perp}^{2}}{z(1-z)}, \quad n=(1-2 z) \frac{M_{X}^{2}}{\left(W^{2}-M_{X}^{2}\right)}
$$

where $m_{q, \perp}^{2}=m_{q}^{2}+k_{\perp}^{2}$ is the transverse quark mass squared. Applying Eq. (2), we get the standard relation for the quark transverse momentum

$$
k_{\perp}^{2}=z(1-z) M_{X}^{2}-m_{q}^{2},
$$

and we consider the light quark mass limit $m_{q} \ll M_{X}^{2}$. The leading contribution to diffractive DIS at HERA comes from light quarks, and from now on we do not distinguish between their masses and account for them by one single mass parameter $m_{q}^{\text {eff }}$.

In the diffractive limit $Q^{2}, M_{X}^{2} \ll W^{2}$, when $z$ and $1-$ $z$ are not very asymmetric, one has with good accuracy:

$$
k_{1}^{0} \simeq k_{1}^{z} \simeq(1-z) \frac{W}{2}, \quad k_{2}^{0} \simeq k_{2}^{z} \simeq z \frac{W}{2},
$$

Let us now define the quark propagators in the photon splitting wave function. Due to the condition $x_{P} \gg$ $x_{2,3}, \ldots$ the soft screening gluons cannot change the longitudinal momenta significantly, but only the transverse momenta. Thus, to calculate the hard part of the amplitude let us first neglect these extra screening gluons. We will show below that adding the extra soft gluons leads only to phase shifts (and their derivatives) in the transverse coordinates, which are going be resummed to all orders in $\alpha_{s}$.

In the chosen frame, the momenta of the exchanged hard and the sum of the soft gluons are

$q_{1} \simeq-x_{P} P^{\prime}+\Delta_{\perp}, \quad q_{2} \simeq \Delta_{\perp}^{\prime}, \quad \delta=\left|\boldsymbol{\Delta}_{\perp}^{\prime}+\boldsymbol{\Delta}_{\perp}\right| \simeq \sqrt{-t}$.

Let us first attach the hard gluon to the lower quark line $k_{2}$. Then the denominator of the quark propagator between the photon and gluon vertices is

$$
\begin{aligned}
\left(k_{2}+q_{1}\right)^{2} & -m_{q}^{2}= \\
& =-z^{2} M_{X}^{2}-z Q^{2} \frac{\beta-x_{P}}{\beta}-\left(k_{\perp}+\Delta_{\perp}\right)^{2}-m_{q}^{2} \\
& \simeq-\frac{\varepsilon^{2}+(1-z)\left(k_{\perp}+\Delta_{\perp}\right)^{2}+z k_{\perp}^{2}}{1-z} \\
& \simeq-\frac{\varepsilon^{2}+\left(k_{\perp}+\Delta_{\perp}\right)^{2}}{1-z}
\end{aligned}
$$

where $\varepsilon^{2}=z(1-z) Q^{2}+m_{q}^{2}$. The first approximation is obtained by substituting $M_{X}^{2}$ from Eq. (3) in the limit $x_{P} \ll \beta$ and the second by using the limit $\Delta_{\perp} \ll k_{\perp}$, realized when $M_{X} \gg m_{q}, \Delta_{\perp}$, giving a result valid at $z \ll 1$.
When the gluon is attached to the upper gluon line, using momentum conservation $q=q^{\prime}+q_{1}+q_{2}$, we get analogously

$$
\left(q-k_{2}^{\prime}\right)^{2}-m_{q}^{2} \simeq-\frac{\varepsilon^{2}+\left(k_{\perp}-\Delta_{\perp}\right)^{2}}{z},
$$

which is strictly valid at $1-z \ll 1$. It is equal to Eq. (5) with the exchanges $z \leftrightarrow(1-z)$ and $k_{\perp} \leftrightarrow-k_{\perp}$.

The expressions (5) and (6) will be used for all values of $z$, as is common practice [5]. This is justified in our asymmetric case $x_{P} \gg x^{\prime}$ because the dominating contribution to the amplitude comes from the configuration that either the quark or the antiquark from the photon is essentially on-shell, and the other carries the negative virtuality of the photon and then absorbs the hard gluon with momentum fraction $x_{P}$ to become essentially onshell.

In the limit considered, $\Delta_{\perp} \ll k_{\perp}$, the quark virtuality $k^{2}$ is conventionally utilized as the factorization scale $\mu_{F}^{2}$ of the process, and is expressed in terms of the energy $\varepsilon$ and the transverse momentum $k_{\perp}$ as

$$
\mu_{F}^{2} \equiv \varepsilon^{2}+k_{\perp}^{2}=z(1-z)\left(M_{X}^{2}+Q^{2}\right) .
$$

Thus, the hard scale depends on both $Q^{2}$ from the spacelike photon and $M_{X}^{2}$ from the time-like final state $X$. Since these can have any values, the QCD factorization is complicated and the physics may be different in the three cases $M_{X} \ll Q, M_{X} \sim Q$ and $M_{X} \gg Q$. The quark propagator (with the hard scale $\mu_{F}^{2}$ in Eq. (7)) is antisymmetric, $k^{2} \leftrightarrow-k^{2}$, with respect to reflection between the space-like and time-like regimes, i.e., with respect to the interchange $M_{X}^{2} \leftrightarrow-Q^{2}$ (or $\varepsilon^{2} \leftrightarrow-k_{\perp}^{2}$ ) as it should be.

The next step is to compute the bilinear spinor combinations $\bar{u}\left(k_{2}, \lambda_{q}\right) \notin\left(\lambda_{\gamma}\right) v\left(k_{1}, \lambda_{\bar{q}}\right)$ in the photon splitting $\gamma^{*} \rightarrow q \bar{q}$. The photon polarization vectors in the $X P^{\prime}$ c.m.s. frame have the following general form

$$
\begin{aligned}
& \varepsilon_{\mu}^{T}\left(\lambda_{\gamma}= \pm 1\right)=\frac{1}{\sqrt{2}}(0,1, \pm i, 0), \\
& \varepsilon_{\mu}^{L}\left(\lambda_{\gamma}=0\right)=\frac{i}{2 W Q}\left(W^{2}+Q^{2}, 0,0, W^{2}-Q^{2}\right) .
\end{aligned}
$$

In particular, by straightforward calculation for the longitudinally (L) polarized photon we simply get the following expression

$$
\bar{u}_{ \pm}\left(k_{2}\right) \notin\left(\lambda_{\gamma}=0\right) v_{\mp}\left(k_{1}\right) \simeq i \sqrt{z(1-z)} Q .
$$

which is not dependent on the transverse momenta of the initial quark and antiquark.

The transversely polarized case requires a separate discussion. Within the dipole picture the diffractive DIS process can be basically decomposed into time-ordered stages. First, the space-like photon with $q^{2}=-Q^{2}$ fluctuates into a $q \bar{q}$ pair, which is then scattered off the target through hard gluon exchange, making the $q \bar{q}$-system time-like, and finally the on-shell quark and antiquark 
scatter softly off the color background field in the proton resulting in a color singlet $X$-system with invariant mass $M_{X}$. Initially, at the moment of the photon fluctuation, the only hard scale is $Q^{2}$, and the transverse momentum of a produced quark is expressed through this scale as $k_{\perp}^{\prime} \simeq \sqrt{z(1-z)} Q$, which is different from the transverse momentum $k_{\perp}$ of a quark in the final state defined in Eq. (44). In particular, the difference between $k_{\perp}$ and $k_{\perp}^{\prime}$ can depend on the actual momentum transfer $\Delta_{\perp}$ for the hard gluon and on the sum of the screening gluons, because according to Eq. (4) the small variation in $k_{\perp}$ due to the attached $\Delta_{\perp}$ brings a significant change in $M_{X}$ for small $z$. The relative coefficient between $k_{\perp}$ and $k_{\perp}^{\prime}$ is $Q / M_{X}$, and has to be taken into account when expressing the transversely polarized photon splitting wave function through the final state transverse momenta. This physical argument agrees well with the kinematics correspond-

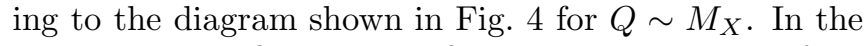
opposite limits $Q \gg M_{X}$ or $Q \ll M_{X}$ the emission of extra gluons significantly complicates the kinematics, and this will be considered in detail below.

As a final result, for the transversely $(\mathrm{T})$ polarized photon, we get in the chiral limit

$$
\bar{u}_{ \pm}\left(k_{2}\right) \notin\left(\lambda_{\gamma}= \pm 1\right) v_{\mp}\left(k_{1}\right)=\frac{Q}{M_{X}} \sqrt{\frac{2 z}{1-z}}\left(k_{1}^{x} \pm i k_{1}^{y}\right) .
$$

The spinor signs \pm here stand for quark/antiquark chiralities, which coincide with the helicity for a quark and have the opposite sign of the helicity for an antiquark. We do not take into account the "++" and "--" components since they are small in the Bjorken limit and for relatively light quark and antiquark.

\section{GENERALIZED UNINTEGRATED GLUON DISTRIBUTIONS}

Before calculating the amplitude for the hard and soft gluon exchanges, we note that the exchanged gluons all originate from the proton color field and should therefore be treated through a common description of a general gluon density. The first, hard gluon carries the dynamics through the longitudinal momentum $x_{P} P$, whereas the soft rescattering gluons carry small momenta $x_{i}^{\prime} \ll x_{P}$ but may transfer a color octet charge that screens the color of the first gluon resulting in an overall color singlet exchange. In this sense, the sum of all soft exchanges acts as a single effective gluon exchange between the $q \bar{q}$ dipole and the proton remnant (see Fig. 5).

As an appropriate description of the density of hard and soft effective gluons, we use the framework of generalized off-diagonal unintegrated gluon distribution functions (UGDF), which naturally appear in the $k_{\perp}$ factorization approach [20]. Within this framework the coupling to a quark is replaced by an off-diagonal UGDF $\mathcal{F}_{g}^{\text {off }}\left(x_{P}, x^{\prime}, \Delta_{\perp}^{2}, \Delta_{\perp}^{\prime}{ }^{2}, \mu_{F}^{2}\right)$, absorbing a factor $C_{F} \alpha_{s}\left(\mu_{\text {soft }}^{2}\right) / \pi$ and by convention also a gluon propagator $\Delta_{\perp}^{-2}$ in order to keep the UGDF regular as $\Delta_{\perp}^{2} \rightarrow 0$.

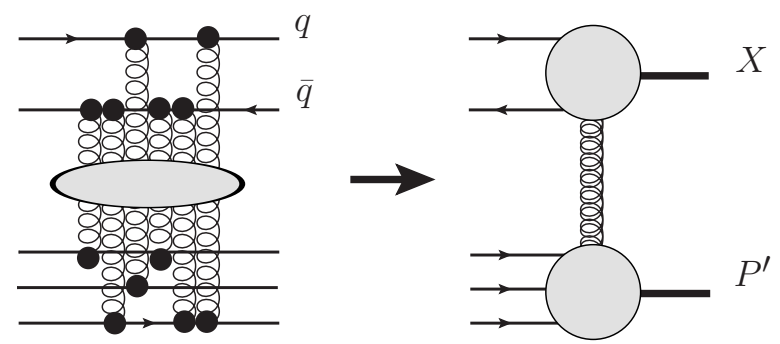

FIG. 5: The sum of the multiple soft gluon exchanges can in an effective description be seen as a single gluon exchange between the $q \bar{q}$ dipole and the color octet proton remnant, resulting in the color singlet $X$-system and a proton in the final state.

The absorbed coupling $\alpha_{s}\left(\mu_{\text {soft }}^{2}\right)$ corresponds to the coupling of a screening gluon with virtuality $\mu_{\text {soft }}^{2} \sim \Delta_{\perp}^{2}$ to a quark in the proton, whereas the coupling of the hard gluon to the $q \bar{q}$ dipole is purely perturbative and occurs at the hard scale $\mu_{F}$.

Generalized parton distributions (GPD) are not very constrained by data. We use a prescription for the generalized UGDF [21], which works well in the description of CDF data on central exclusive charmonium production [22]. This prescription is motivated by positivity constraints for the collinear GPDs 23] and can be considered as a saturation of the Cauchy-Schwarz inequality for the density matrix [24]. It incorporates the dependence on the longitudinal momentum fraction $x^{\prime}$ and the transverse momenta of the soft gluons in an explicitly symmetric way,

$$
\mathcal{F}_{g}^{\text {off }} \simeq \sqrt{\mathcal{F}_{g}\left(x_{P}, \Delta_{\perp}^{2}, \mu_{F}^{2}\right) \mathcal{F}_{g}\left(x^{\prime}, \Delta_{\perp}^{\prime}{ }^{2}, \mu_{\text {soft }}^{2}\right)},
$$

with $\Delta_{\perp} \sim \Delta_{\perp}^{\prime}$. Here $\mathcal{F}_{g}$ is the normal diagonal UGDF, which depends on the gluon virtuality $\Delta_{\perp}^{2}$ and reduces to the well-known collinear gluon PDF $g\left(x, \mu^{2}\right)$ when integrated over this virtuality.

This model (10) is a factorization of the generalized UGDF into a hard part depending on a hard scale $\mu_{F}$ and on $x_{P}$, thus describing the hard gluon coupling to the proton, and a soft part defined at some soft scale $\mu_{\text {soft }}$ and small $x^{\prime} \ll x_{P}$ corresponding to a number of soft gluon couplings. As we will see below, together with the factorization in transverse momentum space, the model (10) provides a QCD factorization of the diffractive amplitude in momentum space.

The UGDF $\mathcal{F}_{g}\left(x, \Delta_{\perp}^{2}, \mu^{2}\right)$ introduced above depends on the gluon virtuality, and this dependence is not theoretically well-known for small virtualities. The UGDF is here modeled using the collinear gluon density $x_{P} g\left(x_{P}, \mu_{F}^{2}\right)$, fixed at the QCD factorization scale $\mu_{F}$, together with a simple Gaussian Ansatz for the dependence on the gluon virtuality $\Delta_{\perp}^{2}$ as

$$
\begin{gathered}
\sqrt{x_{P}} \mathcal{F}_{g}^{\text {off }} \simeq \sqrt{x_{P} g\left(x_{P}, \mu_{F}^{2}\right) x^{\prime} g\left(x^{\prime}, \mu_{\mathrm{soft}}^{2}\right)} f_{G}\left(\Delta_{\perp}^{2}\right), \\
f_{G}\left(\Delta_{\perp}^{2}\right)=1 /\left(2 \pi \rho_{0}^{2}\right) \exp \left(-\Delta_{\perp}^{2} / 2 \rho_{0}^{2}\right)
\end{gathered}
$$


where the Gaussian width $\rho_{0}$ is the soft hadronic scale. As demonstrated below, this scale corresponds to the transverse proton size $r_{p} \sim 1 / \rho_{0}$. The Gaussian smearing is then interpreted as the result of many soft interactions in the bound state proton. The factor $\sqrt{x_{P}}$ in Eq. (11) is absorbed from the hard subprocess part describing the coupling of a hard gluon to the $q \bar{q}$ dipole, and gives us a hint that the off-diagonal UGDF should be proportional to $\sim \sqrt{x_{P} g\left(x_{P}\right)}$.

It is known that at some soft scale $\mu_{\text {soft }} \sim \Lambda_{\mathrm{QCD}}$ collinear PDFs like GRV [25] saturate at small $x^{\prime} \ll x_{P}$, so one can introduce a function $\bar{R}_{g}\left(x^{\prime}, \mu_{\text {soft }}^{2}\right)$, which is assumed to be slowly dependent on $x^{\prime}$ in the case $x^{\prime} \ll x_{P}$ :

$$
\sqrt{x_{P}} \mathcal{F}_{g}^{\text {off }} \simeq \bar{R}_{g}\left(x^{\prime}, \mu_{\text {soft }}^{2}\right) \sqrt{x_{P} g\left(x_{P}, \mu_{F}^{2}\right)} f_{G}\left(\Delta_{\perp}^{2}\right) .
$$

This factor $\bar{R}_{g}\left(x^{\prime}\right)$ contains all the soft physics related with soft gluon couplings to the proton. It is interpreted as the square root of the gluon density at very small $x^{\prime} \ll$ $x_{P}$ and soft scale $\mu_{\mathrm{soft}}^{2}$. This is a non-perturbative object, which contributes to the overall normalization and can be determined from data. As will be seen below, the prescription (12) is consistent with the HERA data for all available $M_{X}^{2}$ and $Q^{2}$.

There is a debate about what the power of the gluon density in the cross section should be (see [13] and references therein). When squaring the amplitude containing (12), this model leads to a linear dependence of the diffractive cross section on the gluon PDF. This linear dependence is the same as in the SCI model, where it describes both diffractive and non-diffractive events, and provides a continuous transition between the two types of events.

This is in contrast to the quadratic dependence on the gluon density often encountered in two-gluon exchange calculations of DDIS [5]. This arises from another prescription for the off-diagonal UGDF in the asymmetric limit $x^{\prime} \ll x_{P}$ and $\mu_{F}^{2} \gg \Delta_{\perp}^{2}$, which in terms of the diagonal UGDF reads [26]

$$
f_{g}^{\text {off }}\left(x_{P}, x^{\prime}, \Delta_{\perp}^{2}, \Delta_{\perp}^{2}, \mu_{F}^{2}\right) \simeq R_{g}\left(x^{\prime}\right) f_{g}\left(x_{P}, \Delta_{\perp}^{2}, \mu_{F}^{2}\right),
$$

where the skewedness parameter ${ }^{1} R_{g} \simeq 1.2-1.3$ is roughly constant at HERA energies, and gives only a small contribution to an overall normalization uncertainty. The factor $R_{g}$ can be approximately taken into account in this case by rescaling the $x_{P}$ argument in the diagonal UGDF as [27]

$$
f_{g}^{\text {off }}\left(x_{P}, x^{\prime}, \Delta_{\perp}^{2}, \Delta_{\perp}^{2}, \mu_{F}^{2}\right) \simeq f_{g}\left(0.41 x_{P}, \Delta_{\perp}^{2}, \mu_{F}^{2}\right) .
$$

Using the same Gaussian Ansatz for the intrinsic transverse momentum dependence as in Eq. (11), we see that prescription (13) leads to a quadratic dependence of the diffractive structure function on the gluon PDF.

\footnotetext{
${ }^{1}$ Our function $\bar{R}_{g}\left(x^{\prime}\right)$ is an analog of this skewedness factor.
}

The unintegrated gluon density in the form (10) reduces to the diagonal form (13) in the kinematical domain where $x^{\prime} \sim x_{P}$ and the hard gluon is soft, $\mu_{F} \sim$ $\mu_{\text {soft }}$. In this limit there is no QCD factorization, so the hard and soft gluons must be taken into account together on equal footing. This may be the case at low $Q^{2}$ and $M_{X}^{2}$, when a larger contribution to the cross section comes from relatively soft scales $\mu_{F} \lesssim 1 \mathrm{GeV}$, and Eq. (11) reduces to

$$
\sqrt{x_{P}} \mathcal{F}_{g}^{\text {off }} \simeq 0.5 x_{P} g\left(0.5 x_{P}, \mu_{F}^{2}\right) f_{G}\left(\Delta_{\perp}^{2}\right) .
$$

where the factor 0.5 appears from the equal momentum sharing between active and screening gluons, since $x_{P}$ is the sum of all gluon momentum fractions. In this sense, the prescription (10) is more general since it describes both $x^{\prime} \ll x_{P}$ and $x^{\prime} \sim x_{P}$ regimes, and contains prescription (13) as a limiting case.

Eq. (15) also leads to a cross section with the gluon density in the second power. It is similar to the " $R_{g^{-}}$ prescription" (13) (if the Gaussian smearing like in Eq. (11) is adopted), and close to its phenomenological form with rescaled argument (14). This is more conventional in the description of the exclusive processes [5], but is valid only for the symmetric case where the two gluon exchanges carry longitudinal momentum fractions close to each other, $x^{\prime} \sim x_{P}$, and are connected to the same factorization scale $\mu_{F}$. This case can also correspond to the "no-soft-exchange" approximation, when the soft rescattering of the on-shell partons in the final state is not taken into account (then $R_{g}=1$ ).

The above discussion leads to the conclusion that whether it is appropriate to use prescription (12) or (15) depends on what kinematical regime one considers. The problem is that $x^{\prime}$ is not strictly constrained by kinematics. Its order of magnitude should $b^{2}$

$$
x^{\prime} \sim \frac{\Delta_{\perp}^{2}}{z(1-z) W^{2}} \simeq \frac{\mu_{\mathrm{soft}}^{2}}{\mu_{F}^{2}} x_{P}
$$

Thus the $x^{\prime} \ll x_{P}$ regime is realized in the perturbative limit of large factorization scale $\mu_{F}$. In the limit $\mu_{F} \rightarrow$ $\mu_{\text {soft }}$, one instead has $x^{\prime} \rightarrow x_{P}$, as naturally required by matching the prescriptions of Eq. (12) and (15).

To summarize this section, we have formulated a framework for the gluon density needed as input to the calculation of the diffractive cross section. Since this describes soft QCD dynamics in the proton, there are necessarily some uncertainties. The precise HERA data on the diffractive cross section are, however, directly sensitive to this gluon density and may, therefore, be used to obtain new information about the gluon PDF at extremely small $x^{\prime}$ values and at different scales.

\footnotetext{
2 This is similar to central exclusive production in $p p$ collisions, where the screening gluons couple to the triplet/antitriplet charges of the proton remnants, which have predominantly equal momentum sharing $z \sim 1 / 2$, and thus we have $x^{\prime} \sim \Delta_{\perp}^{2} / s$.
} 


\section{LEADING-ORDER QUARK DIPOLE CONTRIBUTION}

We are now fully equipped to derive the amplitude for the dipole-proton interaction.

\section{A. Hard-soft factorization}

The total amplitude for $\gamma^{*} p \rightarrow X p$ is decomposed into longitudinal $(\mathrm{L})$ and transverse $(\mathrm{T})$ parts depending on the photon polarization $\lambda_{\gamma}=0, \pm 1$, and each part can be written as a convolution of the hard and soft subprocess amplitudes based on loop integration and cutting rules.

Starting from the general Sudakov decomposition of the total screening gluon momentum $q_{2}=a q^{\prime}+b P^{\prime}+\Delta_{\perp}$ with $a, b \ll x_{P}$, we can write down the amplitude, for example, for transversely polarized photon as

$$
\begin{aligned}
& M_{\mp \pm}^{\lambda_{\gamma}= \pm} \sim \int \frac{d^{2} \Delta_{\perp}}{(2 \pi)^{2}} d a d b \\
& \times \delta\left(\left(k_{1}+k_{2}+q_{2}\right)^{2}-M_{X}^{2}\right) \delta\left(\left(P^{\prime}-q_{2}\right)^{2}\right) \\
& \times M^{\text {hard }}\left(\Delta_{\perp}^{\prime}\right) M^{\text {soft }}\left(\Delta_{\perp}\right)\left(k_{1}^{x} \pm i k_{1}^{y}\right) .
\end{aligned}
$$

The $\delta$-function product can be rewritten as

$$
\delta(\ldots) \delta(\ldots) \simeq \frac{1}{W^{4}} \delta\left(b-x_{B} \frac{\Delta_{\perp}^{2}}{Q^{2}}\right) \delta\left(a+x_{B} \frac{\Delta_{\perp}^{2}}{Q^{2}}\right),
$$

which takes care of the integrals over $a$ and $b$, leading to

$$
\begin{aligned}
M_{ \pm \mp}^{\lambda_{\gamma}= \pm}=\frac{Q}{M_{X}} \sqrt{\frac{2 z}{1-z} \int \frac{d^{2} \Delta_{\perp}}{(2 \pi)^{2}}} & M^{\text {hard }} M^{\text {soft }} \\
& \times\left(k_{1}^{x} \pm i k_{1}^{y}\right),
\end{aligned}
$$

where, in the frame with $q_{\perp}^{\prime}=0$,

$$
k_{1}^{x} \pm i k_{1}^{y}=-\left(k_{2}^{x} \pm i k_{2}^{y}\right) \equiv k^{x} \pm i k^{y} .
$$

Analogously, the longitudinal contribution is

$$
M_{ \pm \mp}^{\lambda_{\gamma}=0}=i \sqrt{z(1-z)} Q \int \frac{d^{2} \Delta_{\perp}}{(2 \pi)^{2}} M^{\text {hard }} M^{\text {soft }} .
$$

To calculate the Fourier transform of the total amplitude we use the convolution theorem

$$
h(q)=\int f(p) g(q-p) d p \quad \rightarrow \quad \hat{h}(x)=\hat{f}(x) \hat{g}(x)
$$

where $\hat{f}$ denotes the Fourier transform of $f$. In our amplitude this convolution is represented by the integral over $\Delta_{\perp}$, while $\delta=\sqrt{-t}=\left|\Delta_{\perp}^{\prime}+\Delta_{\perp}\right|$ plays the role of $q$. Thus, the inverse transformation over the impact parameter $b$ is

$$
M(\delta) \sim \int d^{2} b e^{-i \boldsymbol{\delta} \mathbf{b}} \hat{M}^{\text {hard }}(\mathbf{b}) \hat{M}^{\text {soft }}(\mathbf{b}) .
$$

leading to factorization of the amplitude in $b$-space as a direct consequence of $k_{\perp}$-factorization in impact parameter space.

\section{B. Hard part}

Consider first the hard gluon coupling to $q$ or $\bar{q}$ shown in Fig. 4 in the rotated frame of reference with $q_{\perp}=\Delta_{\perp}$ and $P_{\perp}=-\Delta_{\perp}$, where $k_{2 \perp}^{\prime}=-k_{1 \perp}^{\prime} \equiv k_{\perp}^{\prime}$. In what follows, we use the relation for quark-gluon vertices in the eikonal approximation

$$
\bar{u}\left(k_{2}^{\prime}+q_{1}\right) \gamma^{\mu} u\left(k_{2}^{\prime}\right) \simeq 2 k_{2}^{\prime \mu}, \quad q_{1} \ll k_{2}^{\prime},
$$

and for the product of the $t$-matrices in the large $N_{c}$ limit we have

$$
t_{i j}^{a} t_{k l}^{a} \simeq T_{F} \delta_{i k} \delta_{j l}
$$

The hard part, describing the two possible couplings of the hard gluon to the $q \bar{q}$ pair, can then be written as

$$
M_{L, T}^{\mathrm{hard}}\left(\Delta_{\perp}, k_{\perp}^{\prime}\right)=\int d^{2} \mathbf{r} d^{2} \mathbf{b} \hat{M}_{L, T}^{\mathrm{hard}}(\mathbf{b}, \mathbf{r}) e^{-i \mathbf{r k}_{\perp}^{\prime}} e^{-i \mathbf{b} \boldsymbol{\Delta}_{\perp}},
$$

where $k_{\perp}^{\prime}$ is the transverse momentum of a quark in the intermediate state (see Fig. 4), and the Fouriertransformed hard amplitudes are given by

$$
\begin{aligned}
\hat{M}_{L}^{\text {hard }} & =i \mathcal{C} \alpha_{s}\left(\mu_{F}^{2}\right) \sqrt{\beta} W^{3} z^{3 / 2}(1-z)^{3 / 2} K_{0}(\varepsilon r) \mathcal{V}(\mathbf{b}, \mathbf{r}), \\
\hat{M}_{T, \pm}^{\text {hard }} & =i \mathcal{C} \alpha_{s}\left(\mu_{F}^{2}\right) \sqrt{\frac{2 \beta}{1-\beta}} \frac{1}{\sqrt{x_{P}}} W^{2} z^{1 / 2}(1-z)^{3 / 2} \\
& \times \varepsilon K_{1}(\varepsilon r) \frac{r_{x} \pm i r_{y}}{r} \mathcal{V}(\mathbf{b}, \mathbf{r})
\end{aligned}
$$

where $\mathcal{C}=8 \pi e_{q} \sqrt{\pi \alpha_{e m}} / N_{c}^{2}$, and $K_{0,1}$ are Bessel functions. The function $\mathcal{V}(\mathbf{b}, \mathbf{r})$ is the gluon density in impact parameter space defined as

$$
\begin{aligned}
\mathcal{V}(\mathbf{b}, \mathbf{r}) & =\frac{1}{\alpha_{s}\left(\mu_{\mathrm{soft}}^{2}\right)} \int \frac{d^{2} \Delta_{\perp}}{(2 \pi)^{2}} \sqrt{x_{P}} \mathcal{F}_{g}^{\mathrm{off}}\left(x_{P}, \Delta_{\perp}^{2}\right) \\
& \times\left\{e^{-i \mathbf{r} \boldsymbol{\Delta}_{\perp}}-e^{i \mathbf{r} \boldsymbol{\Delta}_{\perp}}\right\} e^{i \mathbf{b} \boldsymbol{\Delta}_{\perp}}
\end{aligned}
$$

Here, $\mathcal{F}_{g}^{\text {off }}\left(x_{P}, \Delta_{\perp}^{2}\right)$ is the generalized UGDF, and $\mu_{\text {soft }}^{2}$ the typical soft scale of the process given by the gluon virtuality $\sim \Delta_{\perp}^{2}$. The factor containing $\alpha_{s}\left(\mu_{\text {soft }}^{2}\right)$ is introduced in the normalization of the soft part in order to compensate its absorption into the UGDF (see above). Inserting $\mathcal{F}_{g}^{\text {off }}$ from Eq. (12), we finally get

$$
\begin{aligned}
\mathcal{V}(\mathbf{b}, \mathbf{r}) & =\frac{1}{\alpha_{s}\left(\mu_{\mathrm{soft}}^{2}\right)} \frac{\bar{R}_{g}\left(x^{\prime}\right)}{(2 \pi)^{2}} \sqrt{x_{P} g\left(x_{P}, \mu_{F}^{2}\right)} \\
& \times\left[e^{-\frac{\rho_{0}^{2}}{2}|\mathbf{b}-\mathbf{r}|^{2}}-e^{-\frac{\rho_{0}^{2}}{2}|\mathbf{b}+\mathbf{r}|^{2}}\right] .
\end{aligned}
$$

In the Fourier transformation we assumed slow evolution of the QCD coupling $\alpha_{s}\left(\mu_{\text {soft }}^{2}\right)$, as is the case in the analytic perturbation theory discussed in next section or often assumed by freezing the coupling at very small $\mu_{\text {soft }}^{2}$. Thus, in the Gaussian model (11), the unintegrated gluon density in the impact parameter space $\mathcal{V}(\mathbf{b}, \mathbf{r})$ is factorized into a collinear gluon density multiplied by an $(r, b)$-dependent normal distribution. 


\section{Soft part}

We now turn to the soft subprocess amplitude, which can be calculated order-by-order as follows. The softness of the color-screening gluons with $x_{i}^{\prime} \ll x_{P}$ implies that all intermediate particles are on-shell, and that the dipole size $r$ is not changed during the soft interactions. Cutting the intermediate propagators we have only the phase shifts with the same origin as in Eq. (17), and a dependence on the soft momentum exchanges $\Delta_{i, \perp}^{\prime}$.

In particular, for one and two soft gluon exchanges (in the large $N_{c}$ limit) we obtain

$$
\begin{array}{r}
e^{-i \mathbf{r} \mathbf{k}_{\perp}^{\prime}} M_{1}^{\text {soft }}=\mathcal{A} e^{-i \mathbf{r} \mathbf{k}_{\perp}} \frac{1}{\Delta_{\perp}^{\prime 2}}\left[e^{-i \mathbf{r} \boldsymbol{\Delta}_{\perp}^{\prime}}-1\right] \\
e^{-i \mathbf{r k}_{\perp}^{\prime}} M_{2}^{\text {soft }}=\frac{\mathcal{A}^{2}}{2 !} e^{-i \mathbf{r} \mathbf{k}_{\perp}} \int \frac{d^{2} \Delta_{2 \perp}^{\prime}}{(2 \pi)^{2}} \frac{1}{\Delta_{1 \perp}^{\prime 2} \Delta_{2 \perp}^{\prime 2}} \\
\times\left[e^{-i \mathbf{r} \boldsymbol{\Delta}_{\perp}^{\prime}}-e^{-i \mathbf{r} \boldsymbol{\Delta}_{2 \perp}^{\prime}}-e^{-i \mathbf{r} \boldsymbol{\Delta}_{1 \perp}^{\prime}}+1\right]
\end{array}
$$

where $\Delta_{1 \perp}^{\prime}=\Delta_{\perp}^{\prime}-\Delta_{2 \perp}^{\prime}$ and $\mathcal{A}=2 \pi i C_{F} \alpha_{s}\left(\mu_{\text {soft }}^{2}\right)$ with $C_{F} \simeq T_{F} N_{c}$ in the large $N_{c}$ limit. For example, the NLO gluonic contribution to the soft part $M_{2}^{\text {soft }}$ is represented by the four diagrams shown in Fig. 6.

Fourier transformation with respect to $\boldsymbol{\Delta}_{\perp}^{\prime}$ leads to

$$
\begin{aligned}
e^{-i \mathbf{r k}_{\perp}^{\prime}} \hat{M}_{1}^{\mathrm{soft}} & =e^{-i \mathbf{r} \mathbf{k}_{\perp}} \mathcal{A} \mathcal{W}(\mathbf{b}, \mathbf{r}), \\
e^{-i \mathbf{r k}_{\perp}^{\prime}} \hat{M}_{2}^{\mathrm{soft}} & =e^{-i \mathbf{r} \mathbf{k}_{\perp}} \frac{\mathcal{A}^{2} \mathcal{W}(\mathbf{b}, \mathbf{r})^{2}}{2 !}, \ldots
\end{aligned}
$$

where

$$
\mathcal{W}(\mathbf{b}, \mathbf{r})=\frac{1}{2 \pi} \ln \frac{|\mathbf{b}-\mathbf{r}|}{|\mathbf{b}|}
$$

Continuing this procedure we see that summing over the number of soft gluons in the final state leads to exponentiation in impact parameter space, so that for the total soft subprocess amplitude we finally get

$$
e^{-i \mathbf{r k}_{\perp}^{\prime}} \hat{M}^{\mathrm{soft}}(\mathbf{b}, \mathbf{r})=-e^{-i \mathbf{r k}_{\perp}}\left(1-e^{\mathcal{A} \mathcal{W}(\mathbf{b}, \mathbf{r})}\right) .
$$

A similar expression was previously derived in the case of scalar Abelian gauge theory in Ref. [28]. Note that

\begin{tabular}{|c|c|}
\hline 2 & 2 \\
\hline 多 & 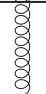 \\
\hline
\end{tabular}
$\hat{M}^{\text {soft }}(\mathbf{b}, \mathbf{r})$ is independent of the photon polarization in the soft limit of small $\Delta_{i, \perp}^{\prime}$.

As mentioned before, the soft gluon exchanges between the final state partons occur at non-perturbatively small
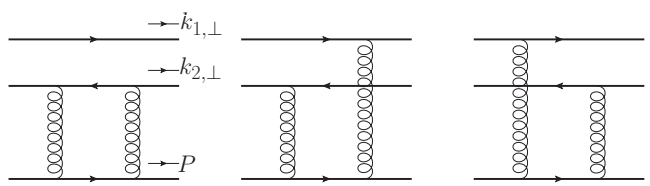

FIG. 6: Illustration of resummation of the gluonic contributions to the (NLO) soft part $M_{2}^{\text {soft }}$ (two gluon exchanges) in the large $N_{c}$ limit. longitudinal $x^{\prime}$ and transverse momentum transfer $\Delta_{\perp}^{\prime}$ at some soft scale $\mu_{\text {soft }}$. The strong coupling $\alpha_{s}\left(\mu_{\text {soft }}^{2}\right)$ is not small in this case. There are several approaches for dealing with the Landau pole at low momentum transfer (see e.g. Ref. [29] and references therein). We use the infrared-finite Analytic Perturbation Theory (APT) 30] approach to parametrize $\alpha_{s}\left(\mu_{\text {soft }}^{2}\right)$ at $\mu_{\text {soft }}^{2} \sim \Lambda_{\mathrm{QCD}}^{2}$. The analytic strong coupling $\alpha_{s}^{\mathrm{APT}}\left(\mu^{2}\right)$ is stable with respect to the choice of the QCD renormalization scheme, higherorder radiative corrections and variations in $\Lambda_{\mathrm{QCD}}$. APT has also proved to give a quantitative description of light quarkonium spectra within the Bethe-Salpeter approach 31] and DIS spin sum rules at low $Q^{2}$ [32].

In the one-loop case, the APT Euclidean function $\mathcal{A}_{1}$, i.e. the analyticized first power of the coupling $\alpha_{s}$ in the Euclidean domain, is 30]

$$
\mathcal{A}_{1}^{(1)}\left(\Delta_{\perp}^{2}\right)=\frac{1}{\beta_{0}}\left[\frac{1}{L}+\frac{\Lambda_{\mathrm{QCD}}^{2}}{\Lambda_{\mathrm{QCD}}^{2}-\Delta_{\perp}^{2}}\right], L=\ln \left(\frac{\Delta_{\perp}^{2}}{\Lambda_{\mathrm{QCD}}^{2}}\right)
$$

where $\beta_{0}$ is the first coefficient of the QCD $\beta$-function.

Since a significant contribution comes from the phase space region with strongly uneven longitudinal momentum distribution between the quark and the antiquark, and where $k_{\perp}$ is not very large, the diffractive structure function becomes sensitive to the model of the strong coupling used to calculate $\alpha_{s}^{\text {soft }}$, and hence to the typical soft scale $\mu_{\text {soft }}$ of the process. To avoid this problem in practice, in the soft regime we are considering, where $\Delta_{\perp} \sim \Lambda_{\mathrm{QCD}}$, we do not extract the value of $\mu_{\mathrm{soft}}$ but rather fix the coupling at $\alpha_{s}^{\text {soft }}=\mathcal{A}_{1}\left(\Lambda_{\mathrm{QCD}}\right) \simeq 0.7$.

Having all the $\mathrm{L}$ and $\mathrm{T}$ amplitudes, we can now finally rotate our frame of reference in the transverse plane to one with $q_{\perp}^{\prime}=0$ and $P_{\perp}^{\prime}=0$, where $k_{\perp}$ is defined as in Eq. (44). In this frame, the variables $b$ and $r$ are the impact parameter and the $q \bar{q}$ dipole size in the final state, respectively.

\section{Diffractive structure function}

Let us turn to the cross section. We are interested in the case when the proton remnant forms a particle in the final state with invariant mass close to the proton mass, so we have a three particle phase space. We may write in general

$$
\begin{aligned}
& q_{0}^{\prime} \frac{d^{3} \sigma^{\gamma^{*} p \rightarrow X p^{\prime}}}{d^{3} \mathbf{q}^{\prime}}=\frac{1}{2 \lambda^{1 / 2}\left(W^{2}, m_{p}^{2},-Q^{2}\right)} \\
& \times \int \frac{d^{3} \mathbf{k}_{1}}{(2 \pi)^{3} 2 k_{1}^{0}} \frac{d^{3} \mathbf{k}_{2}}{(2 \pi)^{3} 2 k_{2}^{0}} \frac{d^{3} \mathbf{P}^{\prime}}{(2 \pi)^{3} 2 P_{0}^{\prime}} q_{0}^{\prime} \delta^{(3)}\left(\mathbf{q}^{\prime}-\mathbf{k}_{1}-\mathbf{k}_{2}\right) \\
& \times(2 \pi)^{4} \delta^{(4)}\left(q+p-k_{1}-k_{2}-P^{\prime}\right) \sum_{\lambda_{q}, \lambda_{\bar{q}}, \lambda_{\gamma}}\left|M_{\lambda_{q} \lambda_{\bar{q}}}^{\lambda_{\gamma}}\right|^{2} .
\end{aligned}
$$

In the large $W$ limit the flux factor is $2 \lambda^{1 / 2}\left(W^{2}, m_{p}^{2},-Q^{2}\right) \simeq 2 W^{2}$. The left hand side 
can be transformed to

$q_{0}^{\prime} \frac{d^{3} \sigma^{\gamma^{*} p \rightarrow X p^{\prime}}}{d^{3} \mathbf{q}^{\prime}} \simeq \frac{W^{2}}{\pi} \frac{d^{2} \sigma^{\gamma^{*} p \rightarrow X p^{\prime}}}{d M_{X}^{2} d t}=-\frac{W^{2} \beta^{2}}{\pi Q^{2}} \frac{d^{2} \sigma^{\gamma^{*} p \rightarrow X p^{\prime}}}{d \beta d t}$

The diffractive structure functions have simple relations to the corresponding differential cross sections

$$
\begin{aligned}
& x_{P} F_{L, T}^{D(4)}\left(\beta, x_{P}, Q^{2}, t\right)=-\frac{Q^{2} \beta}{4 \pi^{2} \alpha_{e m}} \frac{d^{2} \sigma_{L, T}^{\gamma^{*} p \rightarrow X p^{\prime}}}{d \beta d t}, \\
& x_{P} F_{L, T}^{D(3)}\left(\beta, x_{P}, Q^{2}\right) \simeq-\left.\frac{Q^{2} \beta}{4 \pi^{2} \alpha_{e m}} \frac{1}{B_{D}} \frac{d^{2} \sigma_{L, T}^{\gamma^{*} p \rightarrow X p^{\prime}}}{d \beta d t}\right|_{t=0},
\end{aligned}
$$

assuming an exponential $t$-dependence $\sim \exp \left(B_{D} t\right)$ of the cross section on the diffractive slope $B_{D}$. The $\delta$ functions remove the integrals over $\mathbf{P}^{\prime}$ and one of the quark momenta, say, $\mathbf{k}_{1}$, and we get

$$
\begin{gathered}
\frac{d^{2} \sigma^{\gamma^{*} p \rightarrow X p^{\prime}}}{d \beta d t} \simeq-\frac{Q^{2}}{4 \beta^{2}} \frac{\pi}{(2 \pi)^{5}} \frac{1}{W^{6}} \\
\times \int \frac{d^{3} \mathbf{k}_{2}}{z(1-z)} \delta\left(q_{0}+P_{0}-k_{1}^{0}-k_{2}^{0}-P_{0}^{\prime}\right) \sum_{\lambda_{q}, \lambda_{\bar{q}}, \lambda_{\gamma}}\left|M_{\lambda_{q} \lambda_{\bar{q}}}^{\lambda_{\gamma}}\right|^{2}
\end{gathered}
$$

The remaining $\delta$-function removes the integral over $k_{2 z} \simeq$ $z W / 2$. The last phase space integration is rather trivial,

$$
\int d^{2} \mathbf{k}_{\perp}=2 \pi \frac{1}{2} \int_{0}^{k_{\perp, \max }^{2}} d k_{\perp}^{2}=\pi M_{X}^{2} \int_{0}^{\frac{1}{2}} d z(1-2 z) .
$$

Finally, in the full phase space we have to take into account an extra factor of two due to the symmetry with respect to the interchange $z \leftrightarrow 1-z$.

Straightforward calculation leads to the following expressions for the longitudinal and transverse fully-unintegrated diffractive structure functions $F_{L, T}^{D,(4)}\left(x_{P}, Q^{2}, \beta, t\right)$ :

$x_{P} F_{L}^{D(4)}=\mathcal{S} Q^{4} M_{X}^{2} \int_{z_{\text {min }}}^{\frac{1}{2}} d z(1-2 z) z^{2}(1-z)^{2}\left|J_{L}\right|^{2}$

$x_{P} F_{T}^{D(4)}=2 \mathcal{S} Q^{4} \int_{z_{m i n}}^{\frac{1}{2}} d z(1-2 z)\left\{(1-z)^{2}+z^{2}\right\}\left|J_{T}\right|^{2}$,

where $\mathcal{S}=\sum_{q} e_{q}^{2} /\left(2 \pi^{2} N_{c}^{3}\right)$ sums over light quark charges $e_{q}$, and

$$
\begin{array}{r}
J_{L}=i \alpha_{s}\left(\mu_{F}^{2}\right) \int d^{2} \mathbf{r} d^{2} \mathbf{b} e^{-i \boldsymbol{\delta} \mathbf{b}} e^{-i \mathbf{r} \mathbf{k}_{\perp}} K_{0}(\varepsilon r) \\
\times \mathcal{V}(\mathbf{b}, \mathbf{r})\left[1-e^{\mathcal{A W}}\right], \\
J_{T}=i \alpha_{s}\left(\mu_{F}^{2}\right) \int d^{2} \mathbf{r} d^{2} \mathbf{b} e^{-i \boldsymbol{\delta} \mathbf{b}} e^{-i \mathbf{r} \mathbf{k}_{\perp}} \varepsilon K_{1}(\varepsilon r) \\
\times \frac{r_{x} \pm i r_{y}}{r} \mathcal{V}(\mathbf{b}, \mathbf{r})\left[1-e^{\mathcal{A W}}\right]
\end{array}
$$

These are the general expressions of the QCD-based soft multiple gluon rescattering model.

\section{E. Physical interpretation and simplification}

We have now derived Eqs. (24 27), which describe the diffractive structure function. These have nonperturbative soft gluon exchanges as important ingredients, and to calculate these exchanges we have had to make some model assumptions. Some of these assumptions have already been discussed above: we treat the coupling to the quarks using the strong coupling obtained in APT and the coupling to the proton remnant using the function $\bar{R}_{g}$. Moreover, we extrapolate perturbation theory and assume a perturbative propagator for the gluons. The infrared logarithmic divergences in these gluon propagators, which appear at each order in the resummation (see Eqs. (20121)), disappear when the gluon exchanges are resummed to all orders (Eq. (22)).

There is one additional model assumption, as we will explain shortly, but let us first discuss a physical argument based on effective field theory principles, or equivalently, on the uncertainty principle: A gluon with momentum $k$ has a "resolution power," or minimal scale of an object it can resolve, of order $1 / k$. Put another way, physics should not depend on scales much smaller than the resolution scale.

The hard gluon in our calculation can resolve the $q \bar{q}$ dipole with transverse size $r$, allowing us to apply perturbation theory to the hard part. On the other hand, the soft gluons do not carry significant longitudinal momentum fractions, and only small transverse momenta $\Delta_{\perp} \sim \delta=\sqrt{-t}$, so their "maximal resolution" scale is $b \sim 1 / \delta$. This means that the screening gluons cannot dynamically resolve the internal structure of a small $q \bar{q}$ dipole with size $r \ll b$. However, in constructing our model, we extrapolate perturbative QCD to the nonperturbative regime and assume that the soft gluons couple individually to the quark and antiquark, since the essential point of the dynamics here is the color exchange and not the momentum transfer. This is in a similar vein to using quark currents in hadronic matrix elements, such as form factors. The underlying quark and gluon dynamics is still important even at very low scales (see e.g. Ref. [29] for a discussion of this). In this way there is a continuous transition between soft perturbative and soft non-perturbative gluons.

As they stand, the integrals in Eqs. (2627) exhibit unphysical singularities in the angular integrations. This, however, is because of our model assumption, which so far does not fully take into account the resolution power argument. Since the gluons are soft, physics should not depend on the orientation of the $q \bar{q}$ dipole with respect to the impact parameter. This will regulate the unphysical singularities in the angular integration in Eqs. (26]27). This will also allow us to evaluate the integrals analytically, and we will use this in our calculations below. We argue that the resulting expressions, Eqs. (3435) below, are the physically correct expressions for $J_{L}$ and $J_{T}$ to use in Eqs. (2425).

The expression (19) can be considered as a model 
for the unintegrated gluon density in impact parameter space. In particular, it defines the probability to probe a gluon at impact distance $b$ from the proton center with momentum $\Delta_{\perp} \sim 1 / b$ by a hard $q \bar{q}$ dipole with small size $r \ll b$, where the quarks carry the hard momentum $k_{\perp} \sim 1 / r$. The process is considered at a factorization scale equal to the quark virtuality $\mu_{F}^{2}$. The gluons cannot resolve scales below the dipole size $r$. Therefore, the gluon density cannot depend on the orientation of the $q \bar{q}$ dipole with respect to $\mathbf{b}$, i.e., on the angle between $\mathbf{r}$ and b. Also, in this Gaussian model there is no physical reason for an asymmetry of the UGDF with respect to the direction of the vector $\mathbf{b}$. Thus we rewrite our expression (19) in the following way

$$
\mathcal{V} \simeq \frac{1}{\alpha_{s}\left(\mu_{\mathrm{soft}}^{2}\right)} \frac{\bar{R}_{g}\left(x^{\prime}\right)}{2 \pi^{2}} \sqrt{x_{P} g} e^{-\frac{\rho_{0}^{2}}{2}\left(b^{2}+r^{2}\right)} \sinh \left(\rho_{0}^{2} b r\right)
$$

In the small dipole limit $r \ll b$ this becomes

$$
\mathcal{V}(b, r) \simeq \frac{1}{\alpha_{s}\left(\mu_{\mathrm{soft}}^{2}\right)} \bar{R}_{g}\left(x^{\prime}\right) \frac{\rho_{0}^{2}}{2 \pi^{2}} \sqrt{x_{P} g} b r e^{-\frac{\rho_{0}^{2}}{2} b^{2}},
$$

which will be used below to obtain the formula for the DDIS amplitudes.

We can check our formalism by taking the small coupling limit $\alpha_{s}\left(\mu_{\text {soft }}^{2}\right) \sim \alpha_{s}\left(\mu_{F}^{2}\right) \ll 1$, where we can approximate

$$
1-e^{\mathcal{A W}} \simeq-i \alpha_{s}\left(\mu_{\mathrm{soft}}^{2}\right) C_{F} \frac{r}{b}
$$

In the longitudinally polarized case, the Fourier integrals are then reduced to Hankel transforms, leading to

$$
J_{L} \simeq 8 \bar{R}_{g}\left(x^{\prime}\right) \alpha_{s}\left(\mu_{F}^{2}\right) C_{F} \sqrt{x_{P} g} e^{\frac{t}{2 \rho_{0}^{2}}} \frac{\varepsilon^{2}-k_{\perp}^{2}}{\left(\varepsilon^{2}+k_{\perp}^{2}\right)^{3}} .
$$

Thus, in the limit $\alpha_{s} \ll 1$ our model successfully reproduces the standard leading-order two-gluon amplitude [18] and leads to the correct exponential $t$-dependence of the cross-section $\sim \exp \left(B_{D} t\right)$ with diffractive slope $B_{D} \equiv 1 / \rho_{0}^{2}=6.9 \pm 0.2 \mathrm{GeV}^{-2}$ known from HERA data [2]. This gives $\rho_{0} \simeq 380 \mathrm{MeV}$, close to the value of $\Lambda_{\mathrm{QCD}}$. Thus, the Gaussian width $\rho_{0}$ physically corresponds to the effective transverse size of the proton.

However, the strong coupling $\alpha_{s}\left(\mu_{\text {soft }}^{2}\right)$ is not small in the case of small momentum transfers $\lesssim \Lambda_{\mathrm{QCD}}$, and we cannot calculate the integral in $J_{L}$ in general form analytically. The soft phase $\mathcal{A W}$ is not in general small in the Fourier transformation, and in evaluating the Fourier integrals in Eqs. (26127) we should not impose the $\mathcal{W} \ll 1$ condition, but rather keep the $\operatorname{exponent} \exp (\mathcal{A} \mathcal{W})$ with imaginary $\mathcal{A}$. This produces an extra phase shift in the Fourier transform over $\mathbf{r}$, coming from the soft gluon exponentiation in the large $N_{c}$ limit. Employing the "maximum resolution" argument introduced above, we can write

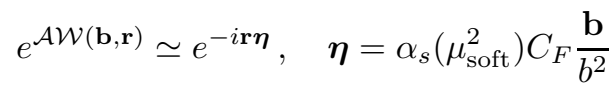

In the longitudinally polarized case the result of the Fourier integration over $\mathbf{r}$ is the Hankel transformation of $K_{0}(\varepsilon r) r$ with respect to the momenta $\mathbf{k}_{\perp}$ and $\mathbf{k}_{\perp}+\boldsymbol{\eta}$. We obtain

$$
\begin{aligned}
& J_{L} \simeq \bar{R}_{g} \frac{\alpha_{s}\left(\mu_{F}^{2}\right)}{\alpha_{s}\left(\mu_{\mathrm{soft}}^{2}\right)} \sqrt{x_{P} g} \frac{\rho_{0}^{2}}{\pi} \int d^{2} \mathbf{b} e^{-i \boldsymbol{\delta} \mathbf{b}} e^{-\frac{1}{2} \rho_{0}^{2} b^{2}} \\
& \times b\left[\frac{2 \varepsilon^{2} E\left(-\frac{k_{\perp}^{2}}{\varepsilon^{2}}\right)-\left(\varepsilon^{2}+k_{\perp}^{2}\right) K\left(-\frac{k_{\perp}^{2}}{\varepsilon^{2}}\right)}{\varepsilon\left(\varepsilon^{2}+k_{\perp}^{2}\right)^{2}}-\right. \\
& \left.\frac{2 \varepsilon^{2} E\left(-\frac{\left(k_{\perp}+\eta\right)^{2}}{\varepsilon^{2}}\right)-\left(\varepsilon^{2}+\left(k_{\perp}+\eta\right)^{2}\right) K\left(-\frac{\left(k_{\perp}+\eta\right)^{2}}{\varepsilon^{2}}\right)}{\varepsilon\left(\varepsilon^{2}+\left(k_{\perp}+\eta\right)^{2}\right)^{2}}\right]
\end{aligned}
$$

in terms of the complete elliptic integrals of the first and second kind, $K(x)$ and $E(x)$, respectively. In the forward limit of small $\delta \ll k_{\perp}$ we expect $k_{\perp} \gg \eta, \eta \equiv|\boldsymbol{\eta}|$.

There is a similar simplification in momentum space for the hard momentum $k_{\perp} \sim 1 / r$, i.e., $\mathbf{k}_{\perp} \boldsymbol{\eta} \sim k_{\perp} \eta$, neglecting the dependence on the direction of the $q \bar{q}$ transverse momentum in the isotropic color field of the proton remnant.

Further, we expand the integrand in Eq. (32) in $\xi=$ $k_{\perp} \eta+\eta^{2} \ll k_{\perp}^{2}$, and keep only the leading term in $\xi$. Taking the last Fourier integral gives

$$
\int d^{2} \mathbf{b} e^{-i \boldsymbol{\delta} \mathbf{b}} e^{-\frac{\rho_{0}^{2}}{2} b^{2}}\left(k_{\perp}+\frac{v}{b}\right)=\frac{2 \pi}{\rho_{0}^{2}} \mathcal{U}(t),
$$

where

$$
\mathcal{U}(t)=k_{\perp} e^{\frac{t}{2 \rho_{0}^{2}}}+\sqrt{\frac{\pi}{2}} v \rho_{0} e^{\frac{t}{4 \rho_{0}^{2}}} I_{0}\left(\frac{-t}{4 \rho_{0}^{2}}\right)
$$

$I_{0}$ is a modified Bessel function, and $v=\alpha_{s}\left(\mu_{\text {soft }}^{2}\right) C_{F}$. The second term is an NLO contribution since it is proportional to the $\alpha_{s}$ in $v$, and typically in the forward limit $t \ll 1 \mathrm{GeV}^{2}$ and in the hard momentum transfer limit it is much smaller than the leading term (we do not consider large $t$, where the whole formalism here does not apply). However, this term is the only leading term which survives in the limit when both $k_{\perp} \rightarrow 0$ and $|t| \sim 1 / b^{2} \rightarrow 0$, so we have to take it into account.

As regards the $t$-dependence, the second term in Eq. (33) decreases at large $t$, but not as rapidly as the first term. To good approximation, the integral over $t$ in the cross section can be written as

$$
\int_{0}^{1} d t \mathcal{U}(t)^{2} \simeq \rho_{0}^{2}\left(k_{\perp}+\sigma_{0} v\right)^{2}, \quad \sigma_{0}=0.73 \mathrm{GeV}
$$

The second term must be taken into account when $k_{\perp}$ is $\lesssim 1 \mathrm{GeV}$.

Straightforward calculation leads to the following re- 
sult for the longitudinal contribution,

$$
\begin{aligned}
& J_{L} \simeq \bar{R}_{g} \sqrt{x_{P} g} \frac{\alpha_{s}\left(\mu_{F}^{2}\right) C_{F} \mathcal{U}(t)}{2 \varepsilon^{3}\left(k_{\perp}^{2}+\varepsilon^{2}\right)^{3}}\left[\left(k_{\perp}^{2}+\varepsilon^{2}\right)\right. \\
& \times\left\{8 K\left(-\frac{k_{\perp}^{2}}{\varepsilon^{2}}\right) \varepsilon^{2}+\pi\left(2{ }_{2} F_{1}\left(\frac{1}{2}, \frac{3}{2} ; 2 ;-\frac{k_{\perp}^{2}}{\varepsilon^{2}}\right) \varepsilon^{2}\right.\right. \\
& \left.\left.\left.+\left(k_{\perp}^{2}+\varepsilon^{2}\right)_{2} F_{1}\left(\frac{3}{2}, \frac{3}{2} ; 2 ;-\frac{k_{\perp}^{2}}{\varepsilon^{2}}\right)\right)\right\}-32 \varepsilon^{4} E\left(-\frac{k_{\perp}^{2}}{\varepsilon^{2}}\right)\right],
\end{aligned}
$$

and for the transverse contribution,

$$
\begin{aligned}
J_{T} \simeq & \bar{R}_{g} \sqrt{x_{P} g} \frac{\alpha_{s}\left(\mu_{F}^{2}\right) C_{F} \mathcal{U}(t)}{2 \varepsilon k_{\perp}^{3}\left(k_{\perp}^{2}+\varepsilon^{2}\right)^{3}} \\
\times & {\left[8\left(\varepsilon^{6}+3 k_{\perp}^{2} \varepsilon^{4}-2 k_{\perp}^{4} \varepsilon^{2}\right) E\left(-\frac{k_{\perp}^{2}}{\varepsilon^{2}}\right)-\left(\varepsilon^{2}+k_{\perp}^{2}\right)\right.} \\
& \times\left\{\pi \left(\left(\varepsilon^{2}-k_{\perp}^{2}\right){ }_{2} F_{1}\left(\frac{1}{2}, \frac{3}{2} ; 2 ;-\frac{k_{\perp}^{2}}{\varepsilon^{2}}\right)\right.\right. \\
& \left.+\left(\varepsilon^{2}+k_{\perp}^{2}\right){ }_{2} F_{1}\left(\frac{3}{2}, \frac{3}{2} ; 2 ;-\frac{k_{\perp}^{2}}{\varepsilon^{2}}\right)\right) k_{\perp}^{2} \\
& \left.\left.+8\left(\varepsilon^{4}+2 k_{\perp}^{2} \varepsilon^{2}\right) K\left(-\frac{k_{\perp}^{2}}{\varepsilon^{2}}\right)\right\}\right]
\end{aligned}
$$

where ${ }_{2} F_{1}$ is the hypergeometric function and $E$ and $K$ are the complete elliptic integrals as above.

\section{GLUON CONTRIBUTION TO THE DIFFRACTIVE STRUCTURE FUNCTION}

In the large- $M_{X}$ limit, gluon emission may be important. In principle, gluons may be radiated from both the $q \bar{q}$ dipole and the hard gluon. The gluons emitted from the quarks are dominantly soft and move collinearly with the quarks, and do not significantly change the invariant mass of the final system $X$. Rather, they dress the quarks to build up their effective mass $m_{q}^{\text {eff }}$, which is, in general, a function of the two hard scales $Q^{2}$ and $M_{X}^{2}$. This mass parameter may be treated as a constituent quark mass. In the current work we do not make predictions for $m_{q}^{\text {eff }}$, but instead extract it from data.

The scale dependence of the effective quark mass in processes with two hard scales like the one under consideration may be complicated. This will be discussed in connection with the numerical results in Sec. VII.

\section{A. Kinematics}

The small $\beta \rightarrow 0$ limit is, therefore, driven by gluon emission from the hard gluon, as illustrated in Fig. 7 The kinematics of the process in the $X P^{\prime}$ c.m.s. frame,

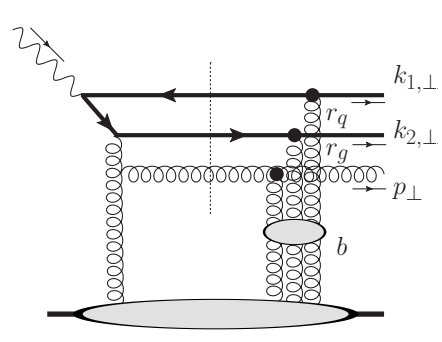

(a)

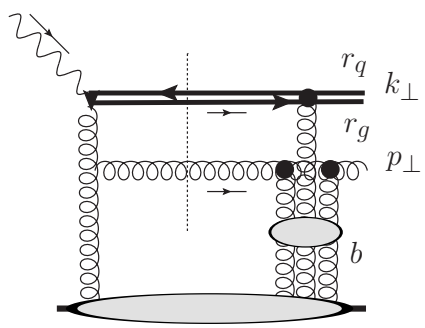

(b)
FIG. 7: Typical diagram for gluon emission in the DDIS final state (a), and its asymptotic limit reducing to the "gluonic dipole" contribution for $\beta \rightarrow 0$ and $p_{\perp} \ll k_{\perp}$ (b).

where $\mathbf{k}_{1, \perp}+\mathbf{k}_{2, \perp}=-\mathbf{p}_{\perp}$, is given by the Sudakov decompositions

$$
\begin{aligned}
& k_{1}=\left(1-z-z^{\prime}\right) q^{\prime}+n_{1} P^{\prime}+k_{1, \perp} \\
& k_{2}=z q^{\prime}+n_{2} P^{\prime}+k_{2, \perp} \\
& p=z^{\prime} q^{\prime}+n_{3} P^{\prime}+p_{\perp}
\end{aligned}
$$

with $n_{1}+n_{2}+n_{3}=0$. Analogously to the $q \bar{q}$ case, we obtain the following expression for the invariant mass $M_{X}$ in terms of momentum fractions $z, z^{\prime}$ :

$$
M_{X}^{2}=\frac{z k_{1, \perp}^{2}+\left(1-z-z^{\prime}\right) k_{2, \perp}^{2}+\left(1-z^{\prime}\right) m_{q}^{2}}{z\left(1-z-z^{\prime}\right)}+\frac{p_{\perp}^{2}}{z^{\prime}} .
$$

$M_{X}$ can also be represented in terms of the invariant mass of the $q \bar{q}$ system as

$$
M_{X}^{2}=\frac{M_{q \bar{q}}^{2}}{1-z^{\prime}}+\frac{p_{\perp}^{2}}{z^{\prime}\left(1-z^{\prime}\right)}, \quad\left(k_{1}+k_{2}\right)^{2}=M_{q \bar{q}}^{2} .
$$

\section{B. Soft gluon resummation and the gluonic dipole limit}

The $q \bar{q} g$-system scatters off the proton by exchanging soft gluons, in the same way as the $q \bar{q}$-system above, and also here the gluon exchanges can be resummed. We have two independent transverse momenta, $k_{1, \perp}^{\prime}$ and $k_{2, \perp}^{\prime}$, outgoing from the hard subprocess, corresponding to impact parameters $r_{1}$ and $r_{2}$. Proceeding as for the $q \bar{q}$ case, we obtain for the $q \bar{q} g$ case

$$
\begin{array}{r}
e^{-i \mathbf{r}_{1} \mathbf{k}_{1, \perp}^{\prime}} e^{-i \mathbf{r}_{2} \mathbf{k}_{2, \perp}^{\prime}} M_{1, q \bar{q} g}^{\mathrm{soft}}=e^{-i \mathbf{r}_{1} \mathbf{k}_{1, \perp}} e^{-i \mathbf{r}_{2} \mathbf{k}_{2, \perp}} \frac{1}{\Delta_{\perp}^{\prime 2}} \\
\times\left[\mathcal{A} e^{-i \mathbf{r}_{1} \boldsymbol{\Delta}_{\perp}^{\prime}}+\mathcal{A} e^{-i \mathbf{r}_{2} \boldsymbol{\Delta}_{\perp}^{\prime}}+\mathcal{A}_{g}\right]
\end{array}
$$

where the prefactor $\mathcal{A}$ was introduced above for the gluon coupling to a $q \bar{q}$ dipole, and $\mathcal{A}_{g}$ corresponds to the case with a gluon coupling to a gluon in the $q \bar{q} g$-system. By explicit calculation of the color factors it can be shown that in the large- $N_{c}$ limit $\mathcal{A}_{g}=-2 \mathcal{A}$. This allows us to perform the Fourier transformation of the soft part over 
$\Delta^{\prime}{ }_{\perp}$ for any number of exchanged gluons and to resum them in the same way, as for $q \bar{q}$ dipole rescattering. For one and two gluon exchanges we have

$$
\begin{aligned}
e^{-i \mathbf{r}_{1} \mathbf{k}_{1, \perp}^{\prime}} e^{-i \mathbf{r}_{2} \mathbf{k}_{2, \perp}^{\prime}} & \hat{M}_{1, q \bar{q} g}^{\mathrm{soft}}=e^{-i \mathbf{r}_{1} \mathbf{k}_{1, \perp}} e^{-i \mathbf{r}_{2} \mathbf{k}_{2, \perp}} \\
& \times \mathcal{A}\left[\mathcal{W}\left(\mathbf{b}, \mathbf{r}_{1}\right)+\mathcal{W}\left(\mathbf{b}, \mathbf{r}_{2}\right)\right], \\
e^{-i \mathbf{r}_{1} \mathbf{k}_{1, \perp}^{\prime}} e^{-i \mathbf{r}_{2} \mathbf{k}_{2, \perp}^{\prime}} & \hat{M}_{2, q \bar{q} g}^{\text {soft }}=e^{-i \mathbf{r}_{1} \mathbf{k}_{1, \perp}} e^{-i \mathbf{r}_{2} \mathbf{k}_{2, \perp}} \\
& \times \frac{\mathcal{A}^{2}}{2 !}\left[\mathcal{W}\left(\mathbf{b}, \mathbf{r}_{1}\right)+\mathcal{W}\left(\mathbf{b}, \mathbf{r}_{2}\right)\right]^{2},
\end{aligned}
$$

where $\mathcal{W}(\mathbf{b}, \mathbf{r})$ is defined above in Eq. (21). Summing over the number of soft gluons in the final state leads to exponentiation in impact parameter space, i.e.,

$$
\begin{aligned}
& e^{-i \mathbf{r}_{1} \mathbf{k}_{1, \perp}^{\prime}} e^{-i \mathbf{r}_{2} \mathbf{k}_{2, \perp}^{\prime}} \hat{M}^{\mathrm{soft}}\left(\mathbf{b}, \mathbf{r}_{1}, \mathbf{r}_{2}\right)= \\
& -e^{-i \mathbf{r}_{1} \mathbf{k}_{1, \perp}} e^{-i \mathbf{r}_{2} \mathbf{k}_{2, \perp}}\left[1-e^{\mathcal{A}\left[\mathcal{W}\left(\mathbf{b}, \mathbf{r}_{1}\right)+\mathcal{W}\left(\mathbf{b}, \mathbf{r}_{2}\right)\right]}\right] .
\end{aligned}
$$

Let us now focus on the leading asymptotic behavior of the diagram in Fig. 7(a) in the limit $\beta \rightarrow 0$. In this limit the hard scale of the process $\mu_{F}^{2} \sim Q^{2} / \beta$ becomes very large. From Eqs. (37) or (38) we see that the $M_{X}^{2} \rightarrow \infty$ limit is realized when $z^{\prime} \ll z$ (more precisely $z^{\prime} \rightarrow 0$ ), so the invariant mass of the $q \bar{q} g \equiv X$ system is

$$
M_{X}^{2} \simeq M_{q \bar{q}}^{2}+\frac{p_{\perp}^{2}}{z^{\prime}} \gg M_{q \bar{q}}^{2},
$$

where

$$
M_{q \bar{q}}^{2} \simeq \frac{k_{\perp}^{2}+m_{q}^{2}}{z(1-z)} \ll M_{X}^{2} .
$$

Consider first the limit where the gluon transverse momentum $p_{\perp}$ is small, such that $\left|\mathbf{k}_{1, \perp}\right| \simeq\left|\mathbf{k}_{2, \perp}\right| \gg\left|\mathbf{p}_{\perp}\right|$. In impact parameter space this kinematical configuration corresponds to the diagram shown in Fig. 7(b). In this limit the $q \bar{q}$ pair is very small, i.e., we have strong ordering in impact parameter space, which can be written as $r_{q} \ll r_{g} \ll b$. In color space the $q \bar{q}$ pair can be considered as a single gluon, and we consider "gluonic dipole" scattering off the target. This is consistent with our expression for the corresponding soft part (39), which in the limit $r_{1} \simeq r_{2} \equiv r_{g}$ reduces to $\sim 1-\exp \left(2 \mathcal{A} \mathcal{W}\left(\mathbf{b}, \mathbf{r}_{g}\right)\right)=1-\exp \left(-\mathcal{A}_{g} \mathcal{W}\left(\mathbf{b}, \mathbf{r}_{g}\right)\right)$, corresponding to the amplitude for soft gluon-gluon scattering. This reproduces the conventional $g g$ dipole result [18] in the small $r_{g} \ll b$ limit, in which the amplitude of the gluonic dipole scattering differs by a factor of $N_{c} / C_{F} \simeq 1 / T_{F}=2$ from the amplitude of the $q \bar{q}$ scattering. Indeed, from our model it follows that in this limit

$$
\begin{aligned}
A_{g g}^{\text {soft }} & =1-\exp \left(-\mathcal{A}_{g} \mathcal{W}\left(\mathbf{b}, \mathbf{r}_{g}\right)\right) \\
& \simeq 2 i \alpha_{s}^{\text {soft }} C_{F} \frac{r}{b}=-2 A_{q \bar{q}}^{\text {soft }}
\end{aligned}
$$

as compared to Eq. (30).

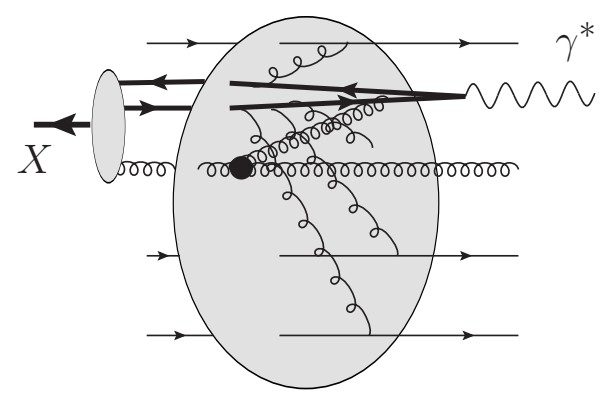

FIG. 8: Illustration of the $q \bar{q} g$ contribution to diffractive DIS.

However, this limiting case cannot give a leading contribution to the diffractive structure function at large $M_{X}$ because of the smallness of the transverse momentum of the final state gluon $p_{\perp} \ll k_{1,2 \perp}$. Due to Eq. (38) the larger gluon $p_{\perp}$, the larger invariant mass $M_{X}$ is produced. At the same time, $p_{\perp}$ can not be significantly larger than the quark and antiquark transverse momenta $k_{1,2 \perp}$. Due to momentum conservation, the maximal $M_{X}$ at fixed $z^{\prime}$ occurs in the limit $p_{\perp} \sim k_{1, \perp} \gg k_{2, \perp}$, which corresponds to $r_{1} \ll r_{2}$ in impact parameter space, leading to $\mathcal{W}\left(\mathbf{b}, \mathbf{r}_{1}\right) \ll \mathcal{W}\left(\mathbf{b}, \mathbf{r}_{2}\right)$. From Eq. (39), this corresponds to the situation when only the $q \bar{q}$ component of the $q \bar{q} g$ system scatters off the target with soft part $A_{q \bar{q}}^{\text {soft }}$. This purely kinematical argument is compatible with an observation [33] with respect to models for parton saturation [34], that the $q \bar{q} g$ and $q \bar{q}$ dipole contributions should saturate to the same value, i.e. $A_{q \bar{q} g}^{\text {soft }} \simeq A_{q \bar{q}}^{\text {soft }}$ at large invariant masses $M_{X}$. In particular, this means that the scattering of the $q \bar{q} g$ system off the proton can not be reduced to the scattering of the $g g$ dipole.

\section{Leading $q \bar{q} g$ contribution to the diffractive structure function}

We argued above that the leading $q \bar{q} g$ contribution to the diffractive structure function in the large $M_{X}$ limit comes from on-shell gluon emission from the hard gluon as in Fig. 7(a). It is clear from Eq. (40) that the relevant limit $M_{X} \rightarrow \infty$ corresponds to essentially on-shell gluon emission with $z^{\prime} \ll z$. The corresponding gluon propagator can be only slightly off-shell to give a leading contribution to the cross section. In this case the $q \bar{q}$ pair takes most of the longitudinal momentum of the $X$ system, and kinematically there is no symmetry with respect to interchange $z^{\prime} \leftrightarrow 1-z^{\prime}$ in such a $q \bar{q} g$ system, whereas for a $q \bar{q}$ dipole this symmetry $z \leftrightarrow 1-z$ holds explicitly. If one allows the active gluon to couple to the $q \bar{q}$ pair directly, the final state gluon connected to the hard quark propagator can not be on-shell, and we get an extra suppression of the cross section. Such a "symmetry breaking" in the $q \bar{q} g$ system does not allow us to reduce it to a symmetric gluonic $g g$ dipole and consider its soft scattering in the same way as $q \bar{q}$ scattering. 
The corresponding physical situation is illustrated in Fig. 8. The hard virtual photon first fluctuates into a virtual $q \bar{q}$ pair, and the leading configuration is when one quark (antiquark) takes most of the photon virtuality whereas the other one is almost on-shell. Then the most virtual quark (antiquark) emits a (less virtual) gluon, which interacts with a slightly virtual sea gluon from the proton background field. This last interaction produces an essentially on-shell final state gluon, which contributes to the final $X$ system. After the first hard gluon exchange both quarks have similar and small virtualities and scatter off the proton background field.

In order to calculate the $q \bar{q} g$ contribution to the diffractive structure function we include a DGLAP splitting of the hard gluon (with longitudinal momentum fraction $x_{P}$ ) into two gluons - one carries momentum fraction $z_{g} x_{P}$ and couples to the hard part, and one is on-shell and contributes to the final state in $\gamma^{*} p$ c.m.s. frame as shown in Fig. 8. The diffractive structure function corresponding to the $q \bar{q} g$ contribution can be then written as (see e.g. 35])

$$
x_{P} F_{q \bar{q} g}^{D(4)} \simeq \frac{1}{N_{c}^{2}} \int \frac{d t_{g} d z_{g}}{t_{g}+m_{g}^{2}} \hat{P}_{g g}\left(z_{g}\right) \frac{\alpha_{s}\left(t_{g}\right)}{2 \pi} x_{P} F_{q \bar{q}}^{D(4)}
$$

where the integral is regulated in the infrared by the effective gluon mass $m_{g} \simeq \Lambda_{\mathrm{QCD}}$ in the gluon propagator. The factor $1 / N_{c}^{2}$ is due to averaging over the color indices (in the large $N_{c}$ limit) of the extra gluon contributing to the color singlet $X$, and $P_{g g}\left(z_{g}\right)$ is the gluon-gluon splitting function

$$
\hat{P}_{g g}(z)=C_{A}\left[\frac{1-z}{z}+\frac{z}{1-z}+z(1-z)\right]
$$

Since the $q \bar{q}$ contribution is dominated by transverse photon polarization, in our formulation the same is true of the $q \bar{q} g$ contribution.

One could also include more gluons in the final state by applying DGLAP evolution of the gluon density, and partially populate the rapidity gap by extra hadronic activity from the hadronization of gluons emitted from the hard gluon in the same way as in Monte Carlo simulations. This would lead to a model describing a smooth transition between diffractive and non-diffractive final states.

Let us finally comment on another approach to resumming multi-gluon exchange, which results in similar eikonal factors $[1-\exp (\ldots)]$ in the amplitudes. This approach was developed by Hautmann, Kunszt, and Soper [36] (HKS) and by Hautmann and Soper [37] (HS), and is applicable in both inclusive and diffractive DIS. This approach is similar to ours, employing factorization and resummation of soft $t$-channel gluons. In diffractive DIS, the incoming partonic dipole is assumed to move closely together in the transverse plane before interacting with the color field of the proton. In the HKS/HS approach all exchanged gluons are treated on the same footing. These gluons collectively carry color singlet charge and are resummed using a Wilson line. In our approach, we use conventional $k_{t}$-factorization in terms of the unintegrated gluon distribution, and we additionally factorize the "hard" gluon, which carries most of the momentum, from the rest of the exchanged gluons, which are much softer $\left(x^{\prime} \ll x_{P}\right)$ and are resummed to all orders. The resummed gluons collectively carry color octet charge, which combined with the first gluon is required to form an overall color singlet exchange. It would be interesting to examine the connections between the two approaches further.

\section{NUMERICAL RESULTS}

The HERA data [2, 3] on DDIS are given in the form of the reduced cross section

$$
x_{P} \sigma_{r}^{D(3)}=x_{P} F_{q \bar{q}, T}^{D(3)}+\frac{2-2 y}{2-2 y+y^{2}} x_{P} F_{q \bar{q}, L}^{D(3)}+x_{P} F_{q \bar{q} g}^{D(3)}
$$

expressed in terms of the diffractive structure functions $F_{L, T}^{D(3)}\left(x_{P}, Q^{2}, \beta\right)$. The momentum transfer $t$ is integrated over since in most of the data the leading proton is not observed, and diffraction is equivalently defined through a large rapidity gap. The kinematical variable $y=Q^{2} /\left(s x_{B}\right) \leq 1$, where $\sqrt{s}=318 \mathrm{GeV}$ is the centerof-mass energy of $e p$-collisions in HERA. In Fig. 9 we compare the latest ZEUS data [2] with the numerical evaluation of our model. A generally very good agreement is found, but this needs to be discussed in detail in order to gain understanding of the dynamics involved.

As discussed in Sec. IV] we need the generalized gluon distribution function in the proton, and use the prescription in Eq. (12) for the UGDF. This reduces the problem to an input of a standard parametrization of the gluon density in the proton, i.e. $x g\left(x, \mu_{F}^{2}\right)$. Here we mainly use the recent CTEQ6L1 parametrization [38], which is in leading order and thereby consistent with our treatment. Below we also consider other parametrizations to illustrate the uncertainty at very small $x$ and factorization scales $\mu_{F}$. The minimum factorization scale $\mu_{F}$ is fixed to be $\mu_{F, \text { min }}^{2}=0.2 \mathrm{GeV}^{2}$ giving rise to a minimum possible fraction of the quark longitudinal momentum $z_{\min }$ in the phase space integral.

The physical parameters that are fixed, are the "soft" coupling $\alpha_{s}^{\text {soft }}=\mathcal{A}_{1}\left(\Lambda_{\mathrm{QCD}}\right) \simeq 0.7$, obtained from infrared-finite analytic perturbation theory (see Sec. $\mathrm{VC}$ ) and used for the coupling of the soft screening gluons, and the gluon mass $m_{g} \simeq \mu_{\text {soft }} \simeq \Lambda_{\mathrm{QCD}}$ adopted as the infrared regulator in the gluon propagator in Eq. (43).

Fixing $\alpha_{s}^{\text {soft }}$ and $m_{g}$, the only free parameters in our model are $m_{q}^{\text {eff }}$ and $\bar{R}_{g}$, representing different soft effects that cannot be calculated or safely estimated. The constituent quark mass $m_{q}^{\text {eff }}$, which enters the kinematics in Sec. III, accounts for the soft gluon radiation from the $q \bar{q}$ dipole and corresponds to forming dressed quarks before hadronization. The soft part $\bar{R}_{g}$ of the off-diagonal UGDF in Eq. (12) can be identified with the square root 


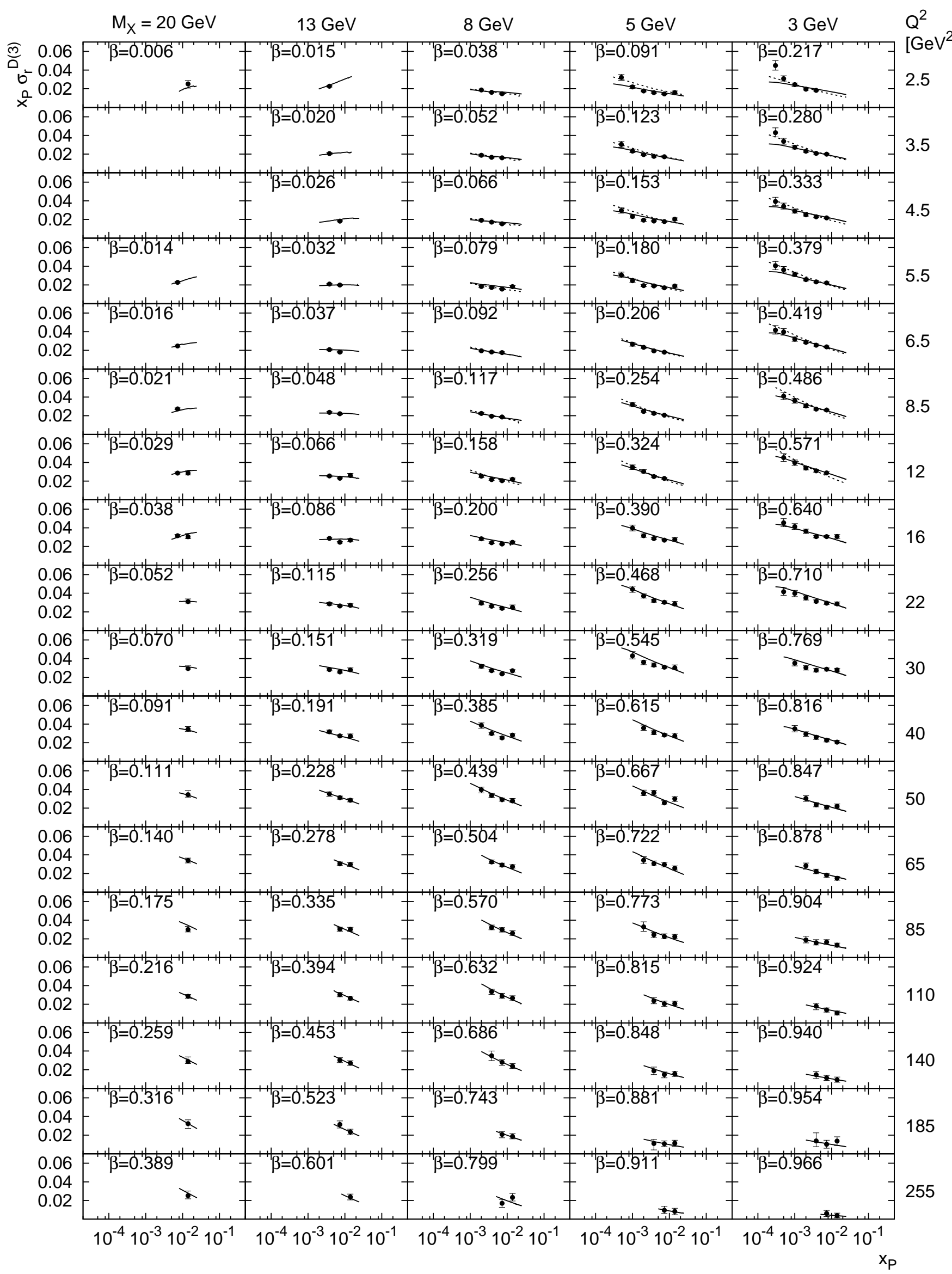

FIG. 9: The reduced cross section $x_{P} \sigma_{r}^{D(3)}\left(x_{P}, \beta, Q^{2}\right)$ as a function of $x_{P}$ for different values of $M_{X}$ and $Q^{2}$. The latest ZEUS data [2], from diffractive deep inelastic scattering events with a large rapidity gap, compared with our model using for the gluon density in the proton the CTEQ6L1 (full line) parametrization [38] and at low $x$ and $Q^{2}$ also the GRV94 (dotted line) parametrization. 
of the "soft" collinear PDF defined at some $x^{\prime} \ll x_{P}$ and $\mu_{\text {soft }}$ which represents the soft scale of the color screening gluons. The sensitivity to these parameters is discussed in the following.

The shapes of the model curves are in quite good agreement with the data, except for a few points at extremely small $x_{P} \lesssim 5 \times 10^{-4}$, and small scales $Q^{2} \lesssim 5 \mathrm{GeV}^{2}$ and $M_{X}$ (see the upper right corner of Fig. $\widetilde{9}$ ). Here, we are in the kinematical domain where the uncertainties in the parametrizations of the gluon density of the proton become extremely large, as illustrated in Fig. 10. As can be seen, for $x \lesssim 10^{-3}$ there are substantial differences between the different gluon parametrizations and the differences become huge for $x \sim 10^{-4}$ and $Q^{2} \lesssim 1$ $\mathrm{GeV}^{2}$. The reason is that there is no data from inclusive DIS or other processes that can measure the gluon density directly in this domain. This gluon PDF uncertainty strongly affects the calculated diffractive structure function at small quark fractions $z$ and/or small $Q^{2}$ and $M_{X}$, where $\mu_{F}$ may drop below $1 \mathrm{GeV}$. In this case $x^{\prime} \sim x_{P}$, due to Eq. (16), so our basic assumptions and QCD factorization itself become less reliable. In principle, the diffractive DIS data can be utilized for selecting the best gluon parametrization among those available in the literature or, even better, for making new gluon parametrizations including the data on DDIS, which depend directly on the gluon density.

Another signature of such uncertainties is the $Q^{2}$ behavior of the soft part of the UGDF, i.e. $\bar{R}_{g}\left(Q^{2}\right)$, which is shown in Fig. 11 for different PDFs. As can be seen, using either the typical leading order CTEQ6L1 PDF 38], which decreases at small $x_{P}$ and $\mu_{F}$, or the more regular but older GRV94HO PDF [25] (see comparison in Fig. 10) to perform the fit of our model to ZEUS data results in quite different fitted $\bar{R}_{g}\left(Q^{2}\right)$. At higher scales, $Q^{2} \gtrsim 16 \mathrm{GeV}^{2}$, the soft factor is quite stable at $\bar{R}_{g} \simeq 1$. However, at $Q^{2} \lesssim 5 \mathrm{GeV}^{2}$, the diffractive cross section calculated with CTEQ6L1 is underestimated by almost an order of magnitude, and in order to get the correct normalization the fitted $\bar{R}_{g}$ value grows significantly. This is mostly because of the strong suppression in CTEQ6L1 at small $x_{P} \lesssim 5 \times 10^{-4}$ at scales $\mu_{F}^{2} \sim 1 \mathrm{GeV}^{2}$. In contrast, the fit with GRV94HO, which does not decrease at small $x_{P}$, leads to a more stable behavior at low $Q^{2}$, such that $\bar{R}_{g}$ essentially becomes an overall normalization constant close to unity.

In order to illustrate how uncertainties in the PDFs and in the UGDF prescriptions affect the $x_{P}$-dependence in comparison with data, we compare our model to the data using both CTEQ6L1 and GRV94HO in the UGDF prescription of Eq. (12). This is our "normal" prescription that gives a linear dependence of the cross section on the gluon density. For comparison we also use CTEQ6L1 with the "old $R_{g}$ "-prescription defined in Eq. (15), which makes the cross section depend on the square of the gluon density. Fig. 12 shows the results in the bins of interest with small scales $M_{X}$ and $Q^{2}$. The "old $R_{g}$ "-prescription leads to an order of magnitude too small diffractive struc- ture function at all $Q^{2}$, which cannot be explained by the expected normalization factor $R_{g}$ of order unity in Eq. (13). The corresponding curves in Fig. 12 have therefore been normalized in order to compare the $x_{P}$-slopes. These slopes are in reasonable agreement with the data, but there is a tendency for a too large curvature generated by the squared gluon density, in particular at large $Q^{2}$.

For the curves with linear gluon density, the curves with GRV94HO lead to better slopes at the smallest $Q^{2}$ and $M_{X}$ than those with CTEQ6L1, but at higher scales they become too steep and cannot describe data. This is not surprising since the old GRV parametrization from 1994 does not take into account later data from HERA and elsewhere, but it provides an interesting alternative due to its more regular behavior at very small $x$ at low scales. The curves fitted with the recent CTEQ6L1 parametrization have better $x_{P}$-slopes at higher scales and this is therefore the main alternative in Fig. 9, in spite of its shortcoming at the very lowest $x_{P}$ points.

The remaining free parameter to discuss is $m_{q}^{\text {eff }}$, the effective mass of the quark and antiquark in the $X$-system which is used in kinematical relations. In Fig. 13 we show fitted values of $m_{q}^{\text {eff }}$ at different scales $M_{X}$ and $Q^{2}$. The diffractive cross section itself is not very sensitive to $m_{q}^{\text {eff }}$, which therefore only varies within the physically reasonable interval $\Lambda_{\mathrm{QCD}} \lesssim m_{q}^{\mathrm{eff}} \lesssim 1.3 \mathrm{GeV}$. Thus, it is mostly of nonperturbative nature and can be interpreted as a constituent quark mass. Nevertheless, $m_{q}^{\text {eff }}$ depends on both $Q^{2}$ and $M_{X}$, indicating that both scales contribute to generating softer gluon radiation. This dependence is, however, non-trivial.

Indeed, a larger invariant mass $M_{X}$ provides a larger phase space, which may accumulate more soft collinear gluons, leading to a larger effective quark mass. On the other hand, a harder photon (large $Q^{2}$ ) can probe a quark at smaller distances, so the $Q^{2}$-dependence of the quark mass obeys renormalization group evolution, i.e. $m_{q}^{\text {eff }}$ should decrease at larger $Q^{2}$. These two effects are indeed observed in the description of data (see Fig. (13) for $M_{X} \lesssim 10 \mathrm{GeV}$. At larger $M_{X}$ the situation changes somewhat due to more hard gluon radiation contributing to $M_{X}$ (in particular, the $q \bar{q} g$ contribution becomes important).

We now investigate the role of the different contributions to $x_{P} \sigma_{r}^{D(3)}$ in Eq. (45)), i.e. $q \bar{q}$ from longitudinally and transversely polarized photons and the $q \bar{q} g$ contribution. In our results shown in Figs. 9 and 12 above, they are all included. We find, however, that the leading order $q \bar{q}$-dipole contribution dominates in all bins of $M_{X}$ and $Q^{2}$ and is enough to describe all data for $\beta \gtrsim 0.2$, below which the $q \bar{q} g$ contribution becomes significant and can be approximated with its leading part calculated via DGLAP splitting of the first, hard gluon.

Fig. 14 shows the $\beta$-spectra for the different contributions. They all vanish in the limits of $\beta \rightarrow 0$, corresponding to large $M_{X}$, and $\beta \rightarrow 1$, corresponding to small or vanishing $M_{X}$ with production of resonances, which is 

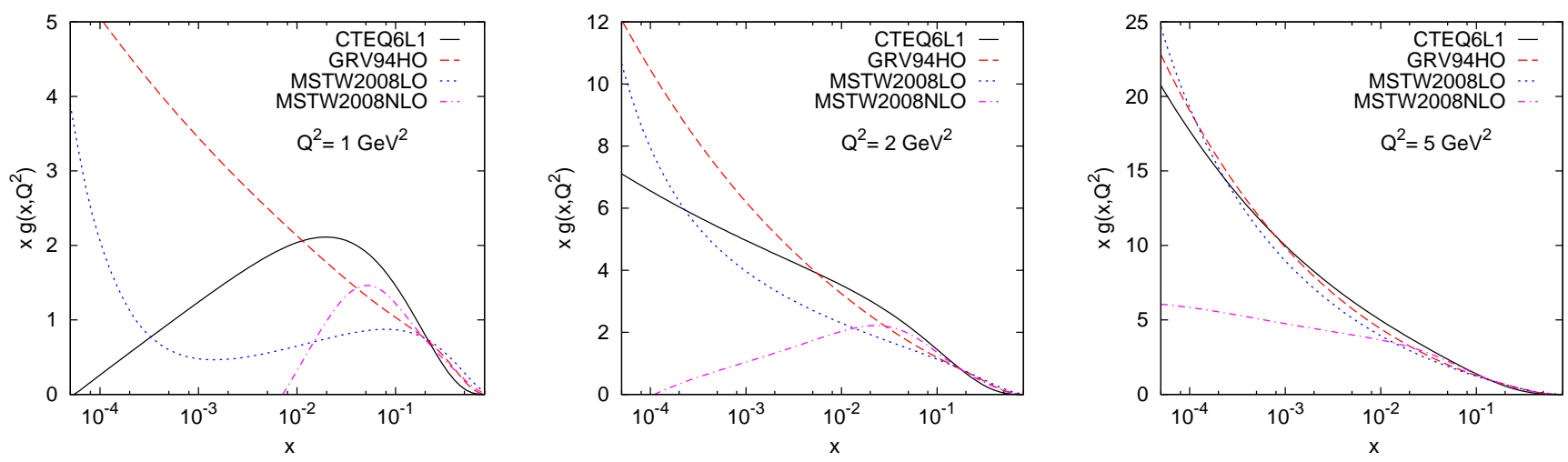

FIG. 10: The dependence on momentum fraction $x$ for the gluon density in the proton at the scales $Q^{2}=1,2$ and 5 GeV ${ }^{2}$ given by the standard parametrizations CTEQ6L1 [38], GRV94HO [25], MSTW2008LO, and MSTW2008NLO [39].

not taken into account here, or no available phase space. The transverse $q \bar{q}$ contribution dominates over the other contributions. The longitudinal $q \bar{q}$ contribution is always small, although it becomes slightly larger at smaller $Q^{2}$ scales. The gluonic $q \bar{q} g$ contribution becomes relatively larger both at high $Q^{2}$ and small $\beta$, where it gives an important contribution that must be taken into account.

\section{CONCLUSIONS AND OUTLOOK}

We have in this paper developed a proper QCD framework for diffractive hard scattering, which contains both hard and soft dynamics. The hard part produces a welldefined state of emerging partons, and the soft part is the rescattering of these partons with the color field of the proton remnant. We have demonstrated that, by taking the Fourier transform from momentum space to impact parameter space, the overall amplitude can be factorized into separate amplitudes for these hard and soft parts. This provides a substantial simplification for the calcula-

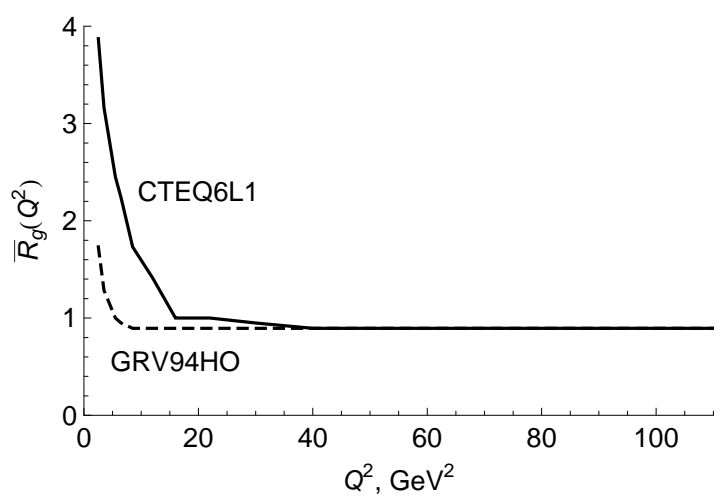

FIG. 11: $Q^{2}$-dependence of the normalization parameter $\bar{R}_{g}$, see Eq. (12), extracted from the HERA data, with solid curve obtained using CTEQ6L1 PDF [38] and dashed curve using GRV94HO PDF [25] for the gluon density in the proton. tion and is consistent with the physical insight that soft, long distance processes cannot affect the hard process occurring on a short distance scale.

The hard part is calculated using perturbative QCD, in the same way as for inclusive DIS. A perturbative hard scale is provided by the photon virtuality $Q^{2}$ and invariant mass $M_{X}$ of the diffractive system, and the process thus occurs at a space-time scale much smaller than the proton size. For small $x$, the hard subprocess $\gamma^{*} g \rightarrow q \bar{q}$ dominates. This process is mediated by a single gluon exchange taking most of the longitudinal momentum transfer, and leaves a proton remnant consisting of the three valence quarks in a color octet state. The proton remnant carries most of the beam momentum, and is therefore well separated in rapidity from the $q \bar{q}$ system.

The soft part of the amplitude accounts for the rescattering of the $q \bar{q}$ pair (in a color octet state) with this remnant. This rescattering is dominated by multiple exchanges of soft gluons, which have larger couplings and less propagator suppression. The result is a negligible change of the momenta of the emerging partons, but an important change of phase is picked up - this is the essence of the eikonal approximation. We find that summing over an arbitrary number of exchanged gluons leads to exponentiation of the soft amplitude, which can be written in a closed analytic form free of infrared divergences. The color exchange, treated in the large- $N_{c}$ approximation, leads to an overall color singlet exchange between the $q \bar{q}$ dipole and the proton. These two color singlet systems then hadronize independently separated by a gap in rapidity as characteristic signature of diffractive scattering.

By invoking physical considerations based on the uncertainty principle, which limits the possible resolution of small momentum transfers, we obtain simplifications of the otherwise complicated angular relations in the impact parameter space. In essence, the orientation of the $q \bar{q}$-dipole relative to the proton color field is physically irrelevant and can be averaged out.

In addition to the leading order contribution from the 


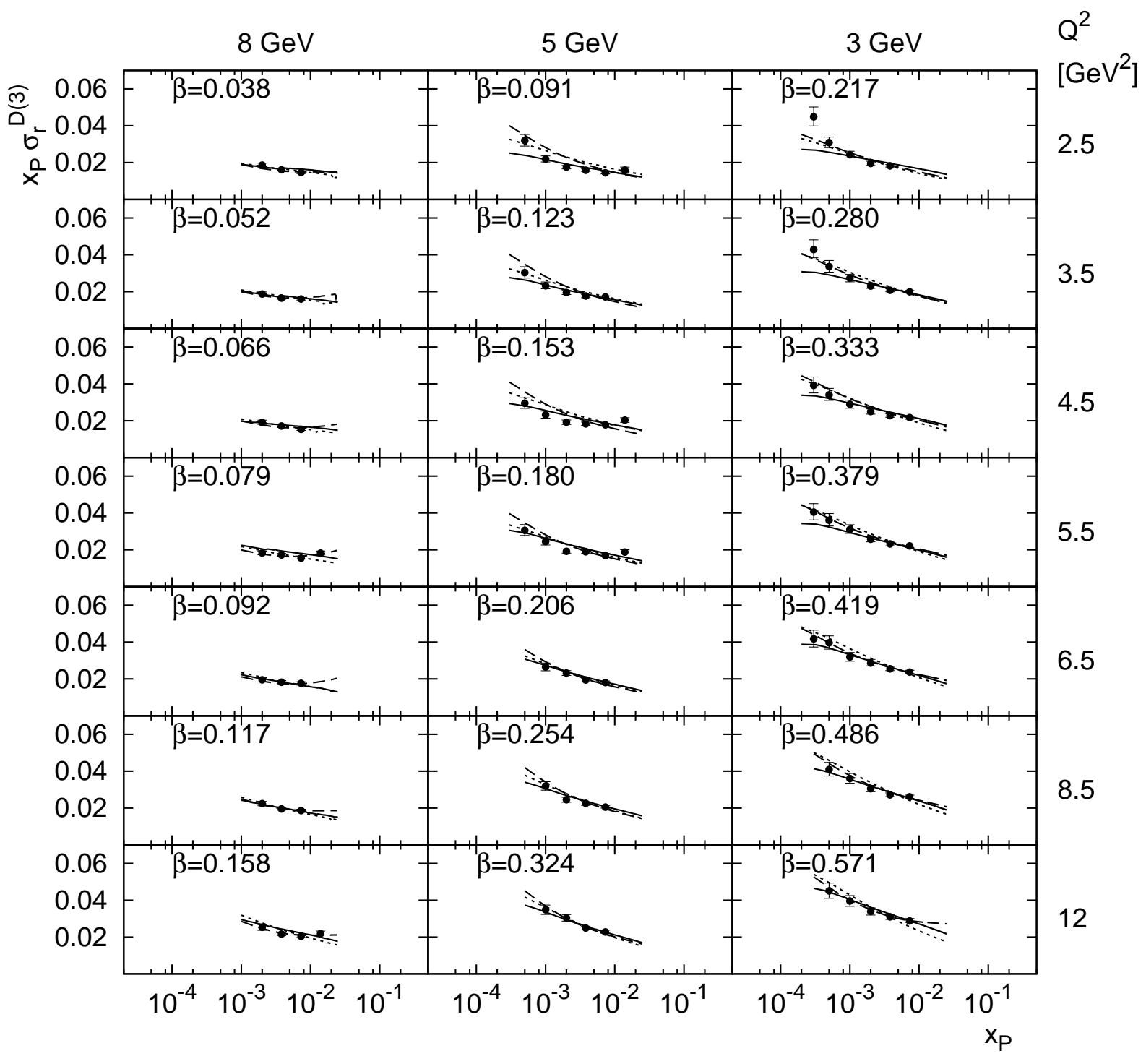

FIG. 12: The reduced cross section $x_{P} \sigma_{r}^{D(3)}\left(x_{P}, \beta, Q^{2}\right)$ as a function of $x_{P}$ in the region of low scales $M_{X} \leq 3,5,8 \mathrm{GeV}$ and $Q^{2}<12 \mathrm{GeV}^{2}$, where the lowest $x_{P}$-values are reached. ZEUS data [2] are compared with the results of the model using CTEQ6L1 (solid line) and GRV94HO (dotted line) in the "square root" UGDF prescription (12), and with CTEQ6L1 in the " $R_{g}$ " prescription (15) (dashed line, normalization adjusted for easy comparison with the other curves).

$q \bar{q}$-dipole, we have also included the next-to-leading contribution $q \bar{q} g$ with an extra gluon in the final state. Here, we find that the most important contribution is emission of this gluon from the exchanged hard gluon (in $\gamma^{*} g \rightarrow q \bar{q}$ ), which can be well approximated by leading logarithmic DGLAP emission.

Numerical evaluation of the analytical results gives good agreement with the precise HERA data on the diffractive deep inelastic cross section. The $q \bar{q}$ contribution is indeed dominant, but at $\beta \lesssim 0.2$, the $q \bar{q} g$ contribution is important. At very small $x_{P} \lesssim 5 \times 10^{-4}$ and scales $\mu_{F}^{2} \sim 1 \mathrm{GeV}^{2}$ the gluon density in the proton, which is used as input in our calculation, is very poorly known and gives a complication in the comparison with the few HERA data points in this extreme region. Stan- dard up-to-date parametrizations have a too low gluon density in this $x, \mu_{F}^{2}$ region, whereas, e.g., the old GRV94 gluon density does better. Since the diffractive cross section depends directly on the gluon density, and not only indirectly via DGLAP evolution as for inclusive DIS, one here obtains an interesting possibility to constrain the gluon density at very small $x$.

Having demonstrated that our theoretical formalism for DDIS does describe HERA data, one may then extract the part describing the multigluon exchange process and apply it to other hard scattering processes. This soft rescattering description ought to be universal, due to the factorization of the hard and soft amplitudes. Thus, one may apply it together with hard processes in $p \bar{p}$ collisions at the Tevatron to describe the different hard diffractive 

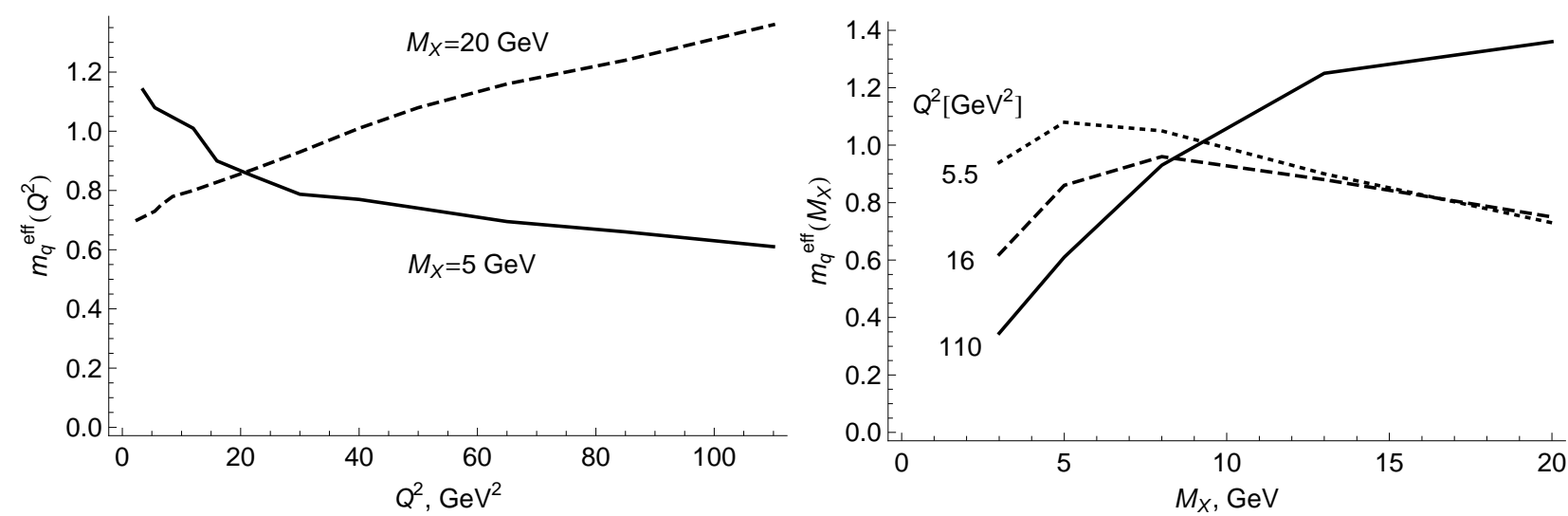

FIG. 13: The effective quark mass parameter $m_{q}^{\text {eff }}$ extracted from the fits of the HERA data shown in Fig. 9, using the CTEQ6L1 gluon parametrization in the "square root" prescription (12). In the left panel, as function of $Q^{2}$ with curves for $M_{X}=20 \mathrm{GeV}$ (dashed) and $M_{X}=5 \mathrm{GeV}$ (solid). In the right panel, as function of $M_{X}$ with curves for $Q^{2}=5.5 \mathrm{GeV}^{2}$ (dotted), $Q^{2}=16 \mathrm{GeV}^{2}$ (dashed) and $Q^{2}=110 \mathrm{GeV}^{2}$ (solid).
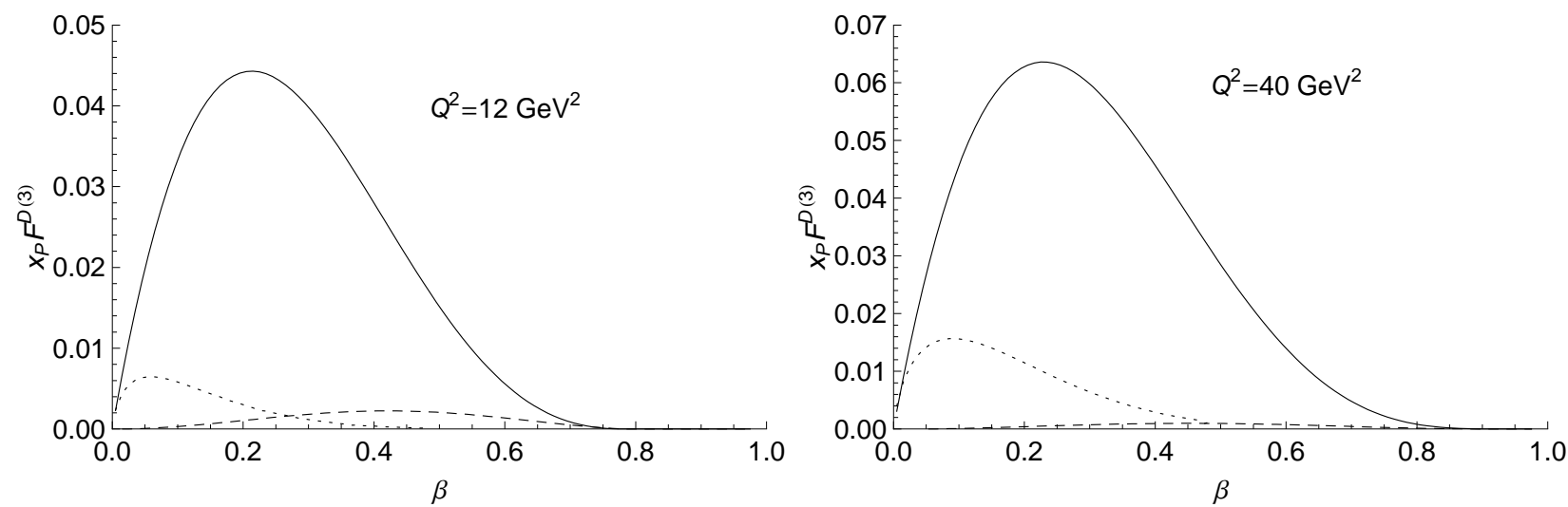

FIG. 14: $\beta$-dependence of the different contributions to the diffractive structure function, Eq. 45), at $Q^{2}=12 \mathrm{GeV}^{2}$ (left) and $Q^{2}=40 \mathrm{GeV}^{2}$ (right). Solid lines represent the transverse part $x_{P} F_{q \bar{q}, T}^{D(3)}$, dashed lines the longitudinal part $x_{P} F_{q \bar{q}, L}^{D(3)}$ and dotted lines the gluonic $q \bar{q} g$ contribution $x_{P} F_{q \bar{q} g}^{D(3)}$. As usual, the prescription of Eq. (12) for the unintegrated gluon density is used with the CTEQ6L1 parametrization. (The quark effective mass is here fixed at $m_{q}^{\text {eff }}=0.9 \mathrm{GeV}$, being the mean value at these scales.)

processed observed there, and then go to the higher energies at the LHC. One may also apply it to more detailed observables in diffractive DIS, such as diffractive dijets or diffractive vector meson production.

However, not only diffractive processes are of interest. The Soft Color Interaction (SCI) model discussed above has previously been successfully applied to both charmonium production and $B$-meson decays [40], and one may expect the model presented in this paper to have interesting applications in such processes too. Moreover, the multi-gluon exchange mechanism will also affect the underlying event, since it effectuates color exchanges that modify the color-string topology and thereby the hadronic final state after hadronization. The underlying event is important in its own right to understand nonperturbative QCD dynamics, and also for understanding of inclusive events when subtracting the Standard Model background in searches for new phenomena at LHC.
Finally we note that in deriving the theoretical formalism presented here, we have not used any assumptions or results from the previous Soft Color Interaction (SCI) model. Our new formalism stands on its own, based on QCD theory and basic physical arguments. The formalism can, however, explain why the simple SCI model has been so successful in describing data on diffractive hard scattering and other phenomena. The assumptions of the SCI model as well as its major features are essentially what comes out as results of the present paper. Of course, our new formalism has a richer dynamical structure and we will therefore attempt to improve the Monte Carlo implementation of the SCI model by replacing its fixed probability for soft gluon exchanges with a mechanism based on the above amplitude for the multiple soft gluon exchanges. This will introduce a non-trivial dependence on the kinematical variables, giving a new level of event-to-event variations. As usual with full event sim- 
ulation using Monte Carlo, this will give access to more detailed studies of both the employed theoretical model and its comparison to data in terms of the indicated more elaborate observables.

\section{Acknowledgments}

This work was supported by the Swedish Research Council and the Carl Trygger Foundation. We are grateful to Igor Anikin for valuable discussions.
[1] A. V. Efremov and A. V. Radyushkin, Phys. Lett. B 94, 245 (1980); Riv. Nuovo Cim. 3N2, 1 (1980); J. C. Collins and D. E. Soper, Nucl. Phys. B 194, 445 (1982); J. C. Collins, D. E. Soper and G. Sterman, Nucl. Phys. B 261, 104 (1985); Nucl. Phys. B 308, 833 (1988); and Phys. Lett. B 438, 184 (1998); G. T. Bodwin, Phys. Rev. D 31, 2616 (1985) [Erratum-ibid. D 34, 3932 (1986)]; J. C. Collins, D. E. Soper and G. Sterman, Adv. Ser. Direct. High Energy Phys. 5, 1 (1988).

[2] S. Chekanov et al. [ZEUS Collaboration], Nucl. Phys. B 816, 1 (2009).

[3] M. Derrick et al., Phys. Lett. B 315, 481 (1993); Phys. Lett. B 346, 399 (1995); T. Ahmed et al., Nucl. Phys. B 429, 477 (1994); Nucl. Phys. B 435, 3 (1995).

[4] A. Hebecker, Phys. Rept. 331, 1 (2000).

[5] M. Wusthoff and A. D. Martin, J. Phys. G 25, R309 (1999).

[6] G. Ingelman, Int. J. Mod. Phys. A 21, 1805 (2006).

[7] R. Bonino et al., Phys. Lett. B 211, 239 (1988).

[8] G. Ingelman and P. E. Schlein, Phys. Lett. B 152, 256 (1985).

[9] A. Edin, G. Ingelman and J. Rathsman, Phys. Lett. B 366, 371 (1996); Z. Phys. C 75, 57 (1997).

[10] G. Ingelman, A. Edin and J. Rathsman, Comput. Phys. Commun. 101, 108 (1997).

[11] B. Andersson, G. Gustafson, G. Ingelman and T. Sjöstrand, Phys. Rept. 97, 31 (1983).

[12] A. Edin, G. Ingelman and J. Rathsman, arXiv:hep-ph/9912539, in proc. 'Monte Carlo generators for HERA physics', DESY-PROC-1999-02 p. 280; R. Enberg, G. Ingelman and N. Tîmneanu, Phys. Rev. D 64, 114015 (2001); A. Edin, G. Ingelman and J. Rathsman, Phys. Rev. D 56, 7317 (1997); D. Eriksson, G. Ingelman and J. Rathsman, Phys. Rev. D 79, 014011 (2009).

[13] S. J. Brodsky, R. Enberg, P. Hoyer and G. Ingelman, Phys. Rev. D 71, 074020 (2005).

[14] R. Pasechnik, R. Enberg and G. Ingelman, arXiv:1004.2912 [hep-ph].

[15] A. H. Mueller, Nucl. Phys. B 335, 115 (1990); N. N. Nikolaev and B. G. Zakharov, Z. Phys. C 49, 607 (1991).

[16] A. Bialas and R. B. Peschanski, Phys. Lett. B 378, 302 (1996); A. Bialas and R. B. Peschanski, Phys. Lett. B 387, 405 (1996); A. Bialas, R. B. Peschanski and C. Royon, Phys. Rev. D 57, 6899 (1998); S. Munier, R. B. Peschanski and C. Royon, Nucl. Phys. B 534, 297 (1998).

[17] N. Nikolaev and B. G. Zakharov, Z. Phys. C 53, 331 (1992).

[18] M. Wusthoff, Phys. Rev. D 56, 4311 (1997).

[19] V. N. Gribov and L. N. Lipatov, Sov. J. Nucl. Phys. 15, 438 (1972); G. Altarelli and G. Parisi, Nucl. Phys. B 126, 298 (1977); Yu. L. Dokshitzer, Sov. Phys. JETP 46, 641 (1977).

[20] S. Catani, M. Ciafaloni and F. Hautmann, Phys. Lett. B
242, 97 (1990);

S. Catani, M. Ciafaloni and F. Hautmann, Nucl. Phys. B 366, 135 (1991);

J. C. Collins and R. K. Ellis, Nucl. Phys. B 360, 3 (1991); L. V. Gribov, E. M. Levin and M. G. Ryskin, Phys. Rept. 100, 1 (1983).

[21] R. S. Pasechnik, A. Szczurek and O. V. Teryaev, Phys. Rev. D 78, 014007 (2008).

[22] R. S. Pasechnik, A. Szczurek and O. V. Teryaev, Phys. Rev. D 81, 034024 (2010).

[23] B. Pire, J. Soffer and O. Teryaev, Eur. Phys. J. C 8, 103 (1999).

[24] X. Artru, M. Elchikh, J. M. Richard, J. Soffer and O. V. Teryaev, Phys. Rept. 470, 1 (2009).

[25] M. Gluck, E. Reya and A. Vogt, Z. Phys. C 67, 433 (1995).

[26] A. G. Shuvaev, K. J. Golec-Biernat, A. D. Martin and M. G. Ryskin, Phys. Rev. D 60, 014015 (1999);

A. D. Martin and M. G. Ryskin, Phys. Rev. D 64, 094017 (2001).

[27] J. R. Cudell, A. Dechambre, O. F. Hernandez and I. P. Ivanov, Eur. Phys. J. C 61, 369 (2009).

[28] S. J. Brodsky et al. Phys. Rev. D 65, 114025 (2002).

[29] S. J. Brodsky, G. F. de Teramond and A. Deur, Phys. Rev. D 81, 096010 (2010).

[30] D. V. Shirkov and I. L. Solovtsov, Phys. Rev. Lett. 79, 1209 (1997).

[31] M. Baldicchi, A. V. Nesterenko, G. M. Prosperi, D. V. Shirkov and C. Simolo, Phys. Rev. Lett. 99, 242001 (2007).

[32] R. S. Pasechnik, D. V. Shirkov, O. V. Teryaev, O. P. Solovtsova and V. L. Khandramai, Phys. Rev. D 81, 016010 (2010).

[33] C. Marquet, Phys. Rev. D 76, 094017 (2007).

[34] K. J. Golec-Biernat and M. Wüsthoff, Phys. Rev. D 59, 014017 (1999); K. J. Golec-Biernat and M. Wüsthoff, Phys. Rev. D 60, 114023 (1999); J. Bartels, K. J. GolecBiernat and H. Kowalski, Phys. Rev. D 66, 014001 (2002); E. Iancu, K. Itakura and S. Munier, Phys. Lett. B 590, 199 (2004).

[35] R. K. Ellis, W. J. Stirling and B. R. Webber, $Q C D$ and collider physics, Cambridge University Press, 1996.

[36] F. Hautmann, Z. Kunszt and D. E. Soper, Phys. Rev. Lett. 81, 3333 (1998); Nucl. Phys. B 563, 153 (1999).

[37] F. Hautmann and D. E. Soper, Phys. Rev. D 63, 011501 (2001); Phys. Rev. D 75, 074020 (2007).

[38] J. Pumplin et al., JHEP 0207, 012 (2002).

[39] A. D. Martin, W. J. Stirling, R. S. Thorne and G. Watt, Eur. Phys. J. C 63, 189 (2009).

[40] A. Edin, G. Ingelman and J. Rathsman, Phys. Rev. D 56, 7317 (1997); C. Brenner Mariotto, M. B. Gay Ducati and G. Ingelman, Eur. Phys. J. C 23, 527 (2002); D. Eriksson, G. Ingelman and J. Rathsman, Phys. Rev. D 79, 014011 (2009). 